TRANSACTIONS OF THE

AMERICAN MATHEMATICAL SOCIETY

Volume 359, Number 5, May 2007, Pages 2191-2220

S 0002-9947(06)04013-X

Article electronically published on December 19, 2006

\title{
ZUCKERMAN FUNCTORS BETWEEN EQUIVARIANT DERIVED CATEGORIES
}

\author{
PAVLE PANDŽIĆ
}

\begin{abstract}
We review the Beilinson-Ginzburg construction of equivariant derived categories of Harish-Chandra modules, and introduce analogues of Zuckerman functors in this setting. They are given by an explicit formula, which works equally well in the case of modules with a given infinitesimal character. This is important if one wants to apply Beilinson-Bernstein localization. We also show how to recover the usual Zuckerman functors from the equivariant ones by passing to cohomology.
\end{abstract}

\section{INTRODUCTION}

One of the basic problems in representation theory is to understand linear representations of a real semisimple connected Lie group $G_{0}$ with finite center. One can instead study related problems of understanding $\mathfrak{g}$-modules, where $\mathfrak{g}$ is the complexified Lie algebra of $G_{0}$, or $(\mathfrak{g}, K)$-modules, where $K$ is the complexification of a maximal compact subgroup of $G_{0}$.

To be more specific, let $\mathcal{M}(\mathfrak{g})$ be the abelian category of modules over $\mathfrak{g}$, or equivalently over the enveloping algebra $\mathcal{U}(\mathfrak{g})$ of $\mathfrak{g}$. Let $K$ be an algebraic group acting on $\mathfrak{g}$ via inner automorphisms, such that the differential of this action identifies the Lie algebra $\mathfrak{k}$ of $K$ with a subalgebra of $\mathfrak{g}$. Then one can define an abelian category $\mathcal{M}(\mathfrak{g}, K)$ of Harish-Chandra modules for the pair $(\mathfrak{g}, K)$. This category consists of $\mathfrak{g}$-modules with an algebraic action of $K$ satisfying the usual compatibility conditions; see $\S 1$. Clearly, there is a natural forgetful functor from $\mathcal{M}(\mathfrak{g}, K)$ into $\mathcal{M}(\mathfrak{g})$.

In the late 1970s, G. Zuckerman proposed a homological construction of HarishChandra modules based on the following observation. Let $T$ be a closed subgroup of $K$. Then there is a natural forgetful functor from $\mathcal{M}(\mathfrak{g}, K)$ into $\mathcal{M}(\mathfrak{g}, T)$. This functor has a right adjoint functor $\Gamma_{K, T}$, called the Zuckerman functor. Roughly speaking, $\Gamma_{K, T}$ attaches to a Harish-Chandra module in $\mathcal{M}(\mathfrak{g}, T)$ its largest HarishChandra submodule for $(\mathfrak{g}, K)$. Zuckerman functors are left exact and usually zero on "interesting" modules. Therefore, one uses the machinery of homological algebra and considers their right derived functors. Using them, one can construct "a lot of" ( $\mathfrak{g}, K)$-modules. For example, if $\mathfrak{g}$ and $K$ are related to $G_{0}$ as above, then all irreducible $(\mathfrak{g}, K)$-modules are submodules of modules obtained in this way for certain specific choices of $(\mathfrak{g}, T)$-modules for various suitable (reductive) $T$. See for example [Vo or [KV]. We review the Zuckerman construction briefly in $\S 1$; a more detailed review is contained in [MP1], $\S 1$.

Received by the editors January 15, 2004 and, in revised form, March 9, 2005.

2000 Mathematics Subject Classification. Primary 22E46.

(C)2006 American Mathematical Society
Reverts to public domain 28 years from publication 
As usual, if one wants to study homological algebra of an abelian category $\mathcal{A}$, the natural setting is the derived category $D(\mathcal{A})$ of $\mathcal{A}$. In our case, one can consider the bounded derived categories $D^{b}(\mathcal{M}(\mathfrak{g}, T))$ and $D^{b}(\mathcal{M}(\mathfrak{g}, K))$ of complexes of HarishChandra modules, the forgetful functor from $D^{b}(\mathcal{M}(\mathfrak{g}, K))$ into $D^{b}(\mathcal{M}(\mathfrak{g}, T))$ and its right adjoint, the derived Zuckerman functor $R \Gamma_{K, T}$ from $D^{b}(\mathcal{M}(\mathfrak{g}, T))$ into $D^{b}(\mathcal{M}(\mathfrak{g}, K))$. One can recover the "classical" derived Zuckerman functors by applying $R \Gamma_{K, T}$ to a single module and then taking cohomology.

On the other hand, a powerful algebro-geometric method of studying $\mathfrak{g}$-modules is the localization theory, due to A. Beilinson and J. Bernstein. Let $\theta$ be a maximal ideal in the center $\mathcal{Z}(\mathfrak{g})$ of the enveloping algebra $\mathcal{U}(\mathfrak{g})$ and denote by $\mathcal{U}_{\theta}$ the quotient of $\mathcal{U}(\mathfrak{g})$ by the two-sided ideal generated by $\theta$. As before, we can define the abelian categories $\mathcal{M}\left(\mathcal{U}_{\theta}\right)$ and $\mathcal{M}\left(\mathcal{U}_{\theta}, K\right)$ of $\mathfrak{g}$-modules, resp. Harish-Chandra modules, with a given infinitesimal character determined by $\theta$. By the Harish-Chandra homomorphism, $\theta$ corresponds to a Weyl group orbit in the linear dual $\mathfrak{h}^{*}$ of a Cartan subalgebra $\mathfrak{h}$ of $\mathfrak{g}$. To each $\lambda$ in $\mathfrak{h}^{*}$, Beilinson and Bernstein attach a sheaf of twisted differential operators $\mathcal{D}_{\lambda}$ on the flag variety $X$ of $\mathfrak{g}$. They prove that the global sections $\Gamma\left(X, \mathcal{D}_{\lambda}\right)$ of $\mathcal{D}_{\lambda}$ are equal to $\mathcal{U}_{\theta}$, and that the higher cohomology vanishes. They define the localization functor from the category $\mathcal{M}\left(\mathcal{U}_{\theta}\right)$ into the category of quasicoherent $\mathcal{D}_{\lambda}$-modules $\mathcal{M}\left(\mathcal{D}_{\lambda}\right)$ on $X$ by $\Delta_{\lambda}(V)=\mathcal{D}_{\lambda} \otimes \mathcal{U}_{\theta} V$. In the case of a regular $\lambda$ which satisfies a geometric positivity condition, the localization functor $\Delta_{\lambda}$ is an equivalence of the category $\mathcal{M}\left(\mathcal{U}_{\theta}\right)$ with $\mathcal{M}\left(\mathcal{D}_{\lambda}\right)$. Its quasi-inverse is the functor of global sections $\Gamma(X,-)$. Under this equivalence, the category $\mathcal{M}\left(\mathcal{U}_{\theta}, K\right)$ of Harish-Chandra modules corresponds to the category $\mathcal{M}\left(\mathcal{D}_{\lambda}, K\right)$ of Harish-Chandra sheaves. Without the positivity condition, the localization functor induces an equivalence of corresponding derived categories $D^{b}\left(\mathcal{M}\left(\mathcal{U}_{\theta}\right)\right)$ and $D^{b}\left(\mathcal{M}\left(\mathcal{D}_{\lambda}\right)\right)$. This enables one to apply the machinery of sheaf cohomology theory to the study of representations. Many results have been obtained using this method, the most famous one being the proof of Kazhdan-Lusztig conjectures.

It is a natural problem to try to connect these two constructions and "localize" the Zuckerman functor construction. The solution is not obvious, as the homological properties of the categories $\mathcal{M}(\mathfrak{g}, K)$ and $\mathcal{M}\left(\mathcal{D}_{\lambda}\right)$ are quite different. Still, Bernstein managed to find a partial solution in the case when $\lambda$ satisfies the geometric positivity condition so that the localization functor is exact. Also, Hecht, Miličić, Schmid and Wolf in their Duality Theorem calculated the cohomology of "standard" Harish-Chandra sheaves on the flag variety in terms of "standard" Zuckerman modules, which can be interpreted as a particular instance of this problem.

In the meantime, the notion of equivariant derived category of Harish-Chandra modules (and D-modules) has been introduced by Beilinson and Ginzburg ( $\mathrm{BB}$, [G]; see also BL2 and [MP1, §2). An analogous construction for equivariant sheaves has been done by Bernstein and Lunts. This category can be used as a bridge between the above two theories. Namely, it allows localization in a natural way, and on the other hand it carries the information about homological algebra of Harish-Chandra modules as well. The reason for the better behavior of this category with respect to localization is the fact that its definition is based on the notion of "weak Harish-Chandra modules." These are defined in the same way as HarishChandra modules, but the compatibility conditions are relaxed: the two actions of $\mathfrak{k}$ are no longer required to agree. In particular, free $\mathcal{U}(\mathfrak{g})$-modules are weak HarishChandra modules for the pair $(\mathfrak{g}, K)$ (but they are not Harish-Chandra modules). 
Similarly, free $\mathcal{U}_{\theta}$-modules are weak $\left(\mathcal{U}_{\theta}, K\right)$-modules, and consequently it is easy to study localization theory for such modules. In $\S 2$ we review the Beilinson-Ginzburg construction, including some details that are usually not mentioned.

A technical complication is that the equivariant derived category of HarishChandra modules $D^{b}(\mathfrak{g}, K)$ is a triangulated category, but not a priori a derived category of any abelian category. Therefore, its use requires some modifications of standard tools used in homological algebra. In particular, a generalization of the notion of derived functors to the case of an arbitrary triangulated category and its localization due to P. Deligne, De is used. This generalized version of derived functors is explained in detail in $[\mathrm{M}]$, Chapter $5, \S 1.3$. In this setting the place of projective and injective resolutions is taken by the notions of $\mathcal{S}$-projective and $\mathcal{S}$-injective objects in the triangulated category in question; here $\mathcal{S}$ is the localizing class defining the localization. These objects were first defined by Verdier in Ve1]; he calls them "free on the left" (respectively right). In the setting of homotopic categories, they were further studied and applied by Spaltenstein in $\mathrm{Sp}$ and Bernstein and Lunts in BL1] and BL2] under the name of K-projectives and K-injectives. One can find an account of this material in [P1], which also contains a summary of some mostly well-known properties of adjoint functors, with emphasis on their use in homological algebra.

$\S 3$ is devoted to the construction of the analogues $\Gamma_{K, T}^{e q}$ of the derived Zuckerman functors in the setting of equivariant derived categories. $\Gamma_{K, T}^{e q}$ is defined as the right adjoint of the forgetful functor from the equivariant derived category $D(\mathfrak{g}, K)$ into $D(\mathfrak{g}, T)$. Analogously, one can define a variant of this functor $\Gamma_{K, T}^{e q}: D\left(\mathcal{U}_{\theta}, T\right) \rightarrow$ $D\left(\mathcal{U}_{\theta}, K\right)$. It is shown how both of them can be described by the same explicit formula, involving the standard complex $N(\mathfrak{k})$ of $\mathfrak{k}$. The main technical result here is that $N(\mathfrak{k})$ is a K-projective equivariant $(\mathfrak{k}, T)$-complex; see $\S 3.2$. Furthermore, it is proved that in the case of $\Gamma_{K, T}^{e q}: D(\mathfrak{g}, T) \rightarrow D(\mathfrak{g}, K)$, when applied to a single Harish-Chandra module $V$, this formula gives a complex with cohomology modules equal to the "classical" derived Zuckerman functors of $V$. In fact, in this case the formula reduces to the Duflo-Vergne formula 1.3.3; see [DV] and [MP1], 1.6. All of the above is also true on the level of the bounded equivariant derived categories.

It is explained in [MP1] how these results can be used to localize the Zuckerman construction.

Finally, let me mention that, as indicated in MP1, one can use localization of the Zuckerman construction to obtain a formula for the cohomology of standard Harish-Chandra sheaves in terms of the derived Zuckerman functors. As a special case one can recover the duality theorem of Hecht, Miličić, Schmid and Wolf from [HMSW]. The details will appear in [MP2].

Most of this paper comes out of my 1995 University of Utah Ph.D. thesis, which was written under guidance of my advisor Dragan Miličić. I am very much indebted to him for his generous help and many suggestions and ideas he supplied me with. I would also like to thank David Vogan for several useful conversations.

\section{Harish-Chandra modules and Zuckerman functors}

1.1. Harish-Chandra pairs and modules. Let $K$ be a complex algebraic group and $\mathfrak{k}$ its Lie algebra. We recall that an algebraic representation of $K$ is a vector space $V$ with a $K$-action, which is a union of $K$-invariant finite-dimensional subspaces $V_{i}$, so that the action on each $V_{i}$ is given by an algebraic group morphism $K \rightarrow \mathrm{GL}\left(V_{i}\right)$. 
Let $\mathcal{A}$ be an associative algebra with identity, with an algebraic action of $K$,

$$
\phi: K \rightarrow \operatorname{Aut}(\mathcal{A}),
$$

and a Lie algebra homomorphism $\psi$ from $\mathfrak{k}$ into $\mathcal{A}$ such that the following conditions hold:

(A1) $\psi$ is $K$-equivariant, i.e.,

$$
\psi \circ \operatorname{Ad} k=\phi(k) \circ \psi \text { for all } k \in K \text {. }
$$

(A2) The differential of the action $\phi$ is the same as the action of $\mathfrak{k}$ on $\mathcal{A}$ given by inner derivations $\psi(\xi), \xi \in \mathfrak{k}$, i.e.,

$$
L(\phi)(\xi)(a)=[\psi(\xi), a]
$$

for any $\xi \in \mathfrak{k}$ and $a \in \mathcal{A}$.

These conditions are related, but in a general situation none of them implies the other.

A pair $(\mathcal{A}, K)$ as above is called a Harish-Chandra pair. The main examples come from a similar notion of a "classical" Harish-Chandra pair, when $\mathcal{A}$ is replaced by a Lie algebra $\mathfrak{g}$. Let $\mathfrak{g}$ be a complex Lie algebra, and $K$ a (usually reductive) algebraic group over $\mathbb{C}$, acting algebraically on $\mathfrak{g}$ via a morphism $\phi: K \rightarrow \operatorname{Int}(\mathfrak{g})$ of algebraic groups. Assume further there is a $K$-equivariant embedding $\psi$ of the Lie algebra $\mathfrak{k}$ of $K$ into $\mathfrak{g}$, such that the differential of $\phi$ is equal to ad $\circ \psi$. Then $(\mathfrak{g}, K)$ is called a (classical) Harish-Chandra pair.

In this situation, $(\mathcal{U}(\mathfrak{g}), K)$ is a Harish-Chandra pair as above: $\phi$ defines an action of $K$ on $\mathcal{U}(\mathfrak{g})$ in an obvious way, and $\psi$ defines an embedding of $\mathfrak{k}$ into $\mathcal{U}(\mathfrak{g})$; the compatibility conditions (A1) and (A2) clearly hold. An important special case of this is when $G$ is a real semisimple Lie group, $\mathfrak{g}$ its complexified Lie algebra, and $K$ the complexification of the maximal compact subgroup of $G$. The map $\phi$ is just the adjoint action and $\psi$ is the inclusion of $\mathfrak{k}$ into $\mathcal{U}(\mathfrak{g})$.

Another set of examples is obtained from a complex Lie group $G$; let $\mathfrak{g}$ be the Lie algebra of $G$, and let $K$ be any algebraic subgroup of $G$, such as a Borel subgroup or its unipotent radical; $K$ can also be $G$ itself. The maps $\phi$ and $\psi$ are, as before, the adjoint action and the inclusion.

In the above cases, when $\mathfrak{g}$ is semisimple, one can also take $\mathcal{A}$ to be the quotient $\mathcal{U}_{\theta}$ of $\mathcal{U}(\mathfrak{g})$ corresponding to an infinitesimal character given by the Weyl group orbit $\theta$ of an element of the dual of a Cartan subalgebra of $\mathfrak{g}$. This is particularly important for us, as it is the setting of the Beilinson-Bernstein localization theory.

Finally, we will occasionally use another simple example: $\mathcal{A}=\mathbb{C}$, and $K$ is an arbitrary algebraic group; $\phi$ is the trivial action on $\mathbb{C}$ and $\psi$ is 0 .

A vector space $V$ is called a weak $(\mathcal{A}, K)$-module or a weak Harish-Chandra module for the pair $(\mathcal{A}, K)$ if:

(HC1) $V$ is an $\mathcal{A}$-module with an action $\pi$;

(HC2) $V$ is an algebraic $K$-module with an action $\nu$;

(HC3) for any $a \in \mathcal{A}$ and $k \in K$ we have

$$
\pi(\phi(k) a)=\nu(k) \pi(a) \nu(k)^{-1} ;
$$

i.e., the $\mathcal{A}$-action map $\mathcal{A} \otimes V \rightarrow V$ is $K$-equivariant.

In the future we will denote the representation $\pi \circ \psi$ of $\mathfrak{k}$ on $V$ simply by $\pi$. This should not create confusion; in fact, in the most important examples $\psi$ is injective and $\mathfrak{k}$ can be identified with a Lie subalgebra of $\mathcal{A}$. 
The action $\nu$ of $K$ differentiates to an action of $\mathfrak{k}$ which we denote also by $\nu$. We put $\omega(\xi)=\nu(\xi)-\pi(\xi)$ for $\xi \in \mathfrak{k}$. The following lemma is [MP1, 2.1].

1.1.1. Lemma. Let $V$ be a weak $(\mathcal{A}, K)$-module. Then $\omega$ is a representation of $\mathfrak{k}$ on $V$, which is $K$-equivariant, i.e., $\omega(\operatorname{Ad}(k) \xi)=\nu(k) \omega(\xi) \nu(k)^{-1}$ for $\xi \in \mathfrak{k}$ and $k \in K$. Moreover, $\omega$ commutes with the action $\pi$ of $\mathcal{A}$, i.e., $[\omega(\xi), \pi(a)]=0$ for $\xi \in \mathfrak{k}$ and $a \in \mathcal{A}$.

We say that a weak $(\mathcal{A}, K)$-module $V$ is an $(\mathcal{A}, K)$-module if $\omega=0$, i.e., if in addition to (HC1)-(HC3) it satisfies:

(HC4) $\pi(\xi)=\nu(\xi)$ for all $\xi \in \mathfrak{k}$.

A morphism $\alpha: V \rightarrow W$ of two weak $(\mathcal{A}, K)$-modules is a linear map which is a morphism for both $\mathcal{A}$ - and $K$-module structures. We denote by $\mathcal{M}(\mathcal{A}, K)_{w}$ the category of all weak $(\mathcal{A}, K)$-modules, and by $\mathcal{M}(\mathcal{A}, K)$ its full subcategory of $(\mathcal{A}, K)$-modules. Clearly, these are abelian categories.

The inclusion functor from $\mathcal{M}(\mathcal{A}, K)$ into $\mathcal{M}(\mathcal{A}, K)_{w}$ has both adjoints. The right (respectively left) adjoint is the functor of taking invariants (respectively coinvariants) with respect to $\omega$, denoted by $U \mapsto U^{\mathfrak{k}}$ (respectively $U \mapsto U_{\mathfrak{k}}$ ). Indeed, 1.1.1 implies that the actions of $\mathcal{A}$ and $K$ on $U$ induce actions on $U^{\mathfrak{k}}$ and $U_{\mathfrak{k}}$. It is clear that $U^{\mathfrak{k}}$ is the largest $(\mathcal{A}, K)$-submodule of $U$, and that $U_{\mathfrak{k}}$ is the largest $(\mathcal{A}, K)$-quotient of $U$. So we get

1.1.2. Proposition. The functors $U \longmapsto U^{\mathfrak{k}}$ and $U \longmapsto U_{\mathfrak{k}}$ are right respectively left adjoint to the forgetful functor from $\mathcal{M}(\mathcal{A}, K)$ into $\mathcal{M}(A, K)_{w}$. In other words, for any $(\mathcal{A}, K)$-module $V$,

$\operatorname{Hom}_{(\mathcal{A}, K)}(V, U)=\operatorname{Hom}_{(\mathcal{A}, K)}\left(V, U^{\mathfrak{k}}\right) \quad$ and $\quad \operatorname{Hom}_{(\mathcal{A}, K)}(U, V)=\operatorname{Hom}_{(\mathcal{A}, K)}\left(U_{\mathfrak{k}}, V\right)$.

1.2. Change of algebras. Let $(\mathcal{A}, K)$ and $(\mathcal{B}, K)$ be two Harish-Chandra pairs. Denote by $\phi_{\mathcal{A}}, \psi_{\mathcal{A}}$ and $\phi_{\mathcal{B}}, \psi_{\mathcal{B}}$ the corresponding maps. Assume that there is a morphism of algebras $\gamma: \mathcal{B} \rightarrow \mathcal{A}$ such that

$$
\gamma \circ \phi_{\mathcal{A}}(k)=\phi_{\mathcal{B}}(k) \circ \gamma, \quad k \in K,
$$

i.e., $\gamma$ is $K$-equivariant. If $V$ is a weak $(\mathcal{A}, K)$-module, we can view it as a weak $(\mathcal{B}, K)$-module, where the action of $\mathcal{B}$ is given by $\tilde{\pi}(b)=\pi(\gamma(b))$ for $b \in \mathcal{B}$. The equivariance condition is preserved because of the equivariance of $\gamma$. This defines an exact functor For $=\operatorname{For}_{\mathcal{B}, \mathcal{A}}$ from the category $\mathcal{M}(\mathcal{A}, K)_{w}$ into the category $\mathcal{M}(\mathcal{B}, K)_{w}$. This forgetful functor has both adjoints, analogous to the well-known functors ind and pro; see for example $[\mathrm{KV}]$. We will need the left adjoint ind, defined as

$$
\operatorname{ind}_{\mathcal{A}, \mathcal{B}}(V)=\mathcal{A} \otimes_{\mathcal{B}} V
$$

for a weak $(\mathcal{B}, K)$-module $V$. Here $\otimes_{\mathcal{B}}$ is taken with respect to the right multiplication on $\mathcal{A}$ and the given action on $V$. $\mathcal{A}$ acts on $\mathcal{A} \otimes_{\mathcal{B}} V$ via left multiplication in the first factor, and $K$ acts by the tensor product of the actions $\phi_{\mathcal{A}}$ on $\mathcal{A}$ and $\nu_{V}$ on $V$. One easily checks that $\operatorname{ind}_{\mathcal{A}, \mathcal{B}}(V)$ is a weak $(\mathcal{A}, K)$-module, and that ind ${ }_{\mathcal{A}, \mathcal{B}}$ 
is a functor which is left adjoint to For. This is just a version of the standard "extension of scalars" construction. So we have

1.2.1. Proposition. Let $\gamma: \mathcal{B} \rightarrow \mathcal{A}$ be a $K$-equivariant morphism of algebras. Then the corresponding forgetful functor For : $\mathcal{M}(\mathcal{A}, K)_{w} \rightarrow \mathcal{M}(\mathcal{B}, K)_{w}$, has a left adjoint, the functor ind ${ }_{\mathcal{A}, \mathcal{B}}$ defined above.

In case $K$ is reductive, we can use this result to construct projectives in the categories $\mathcal{M}(\mathcal{A}, K)_{w}$ and $\mathcal{M}(\mathcal{A}, K)$. Namely, clearly $\mathcal{M}(K)=\mathcal{M}(\mathbb{C}, K)_{w}$ and this category is semisimple, hence all its objects are projective. Furthermore, the inclusion $\mathbb{C} \rightarrow \mathcal{A}$ given by $\lambda \mapsto \lambda 1$ is clearly $K$-equivariant. Since $\operatorname{For}_{\mathbb{C}, \mathcal{A}}$ is exact, its left adjoint ind $\operatorname{A}_{\mathcal{A}, \mathbb{C}}$ preserves projectives. So for any $V \in \mathcal{M}(\mathcal{A}, K)_{w}$, ind $\operatorname{in}_{\mathcal{A}, \mathbb{C}}(V)$ is a projective weak $(\mathcal{A}, K)$-module. On the other hand, the adjunction morphism $\Psi_{V}: \operatorname{ind}_{\mathcal{A}, \mathbb{C}}(V) \rightarrow V$ is surjective, since $\Psi_{V}(1 \otimes v)=v$ for any $v \in V$. So $V$ is a quotient of a projective module.

Using this together with 1.1 .2 , we can similarly prove that $\mathcal{M}(\mathcal{A}, K)$ also has enough projectives. Hence we have proved

1.2.2. Proposition. Let $K$ be a reductive algebraic group. Then the categories $\mathcal{M}(\mathcal{A}, K)_{w}$ and $\mathcal{M}(\mathcal{A}, K)$ have enough projectives.

Let us note that if we in addition assume that $\psi_{\mathcal{A}}=\gamma \circ \psi_{\mathcal{B}}$, then clearly For $_{\mathcal{B}, \mathcal{A}}$ sends $\mathcal{M}(\mathcal{A}, K)$ into $\mathcal{M}(\mathcal{B}, K)$. Moreover, an easy calculation shows that the $\omega$ action on $\operatorname{ind}_{\mathcal{A}, \mathcal{B}} V$ is equal to the tensor product of the trivial action on $\mathcal{A}$ with $\omega_{V}$. It follows that ind $\mathcal{A}_{\mathcal{A}, \mathcal{B}}$ sends $(\mathcal{B}, K)$-modules into $(\mathcal{A}, K)$-modules. This implies the following variant of 1.2.1.

1.2.3. Proposition. Let $\gamma: \mathcal{B} \rightarrow \mathcal{A}$ be a K-equivariant morphism of algebras such that $\psi_{\mathcal{A}}=\gamma \circ \psi_{\mathcal{B}}$. Then the functor $\operatorname{ind}_{\mathcal{A}, \mathcal{B}}: \mathcal{M}(\mathcal{B}, K) \rightarrow \mathcal{M}(\mathcal{A}, K)$ is left adjoint to the forgetful functor from $\mathcal{M}(\mathcal{A}, K)$ into $\mathcal{M}(\mathcal{B}, K)$.

1.3. Change of groups and Zuckerman functors. Let $(\mathcal{A}, K)$ be a HarishChandra pair, with $\phi_{K}: K \rightarrow \operatorname{Aut}(\mathcal{A})$ and $\psi_{K}: \mathfrak{k} \rightarrow \mathcal{A}$ the corresponding morphisms.

Let $T$ be another algebraic group and $\gamma: T \rightarrow K$ a morphism of algebraic groups. The main example is the inclusion of a closed subgroup. We denote the differential of $\gamma$ again by $\gamma$; in applications it will always be injective. It is easily checked that $(\mathcal{A}, T)$ becomes a Harish-Chandra pair if we define $\phi_{T}=\phi_{K} \circ \gamma$ and $\psi_{T}=\psi_{K} \circ \gamma$. Moreover, composing the $K$-action with $\gamma$ to get a $T$-action clearly defines a forgetful functor from $\mathcal{M}(\mathcal{A}, K)_{w}$ into $\mathcal{M}(\mathcal{A}, T)_{w}$, and also from $\mathcal{M}(\mathcal{A}, K)$ into $\mathcal{M}(\mathcal{A}, T)$. We want to describe right adjoints of these forgetful functors.

First we treat the case $T=\{1\}$. Then, as was proved in [MP1, 2.2] (see also [MP1, 1.2]), the right adjoint of For : $\mathcal{M}(\mathcal{A}, K)_{w} \rightarrow \mathcal{M}(\mathcal{A})$ is $\operatorname{Ind}_{w}$, given by

$$
\operatorname{Ind}_{w}(V)=R(K) \otimes V=R(K, V) .
$$

Here $V$ is an $\mathcal{A}$-module, $R(K)$ denotes the space of regular functions on $K$, and $R(K, V)$ denotes the space of $V$-valued regular functions on $K$. Recall that $K$ acts on $\operatorname{Ind}_{w}(V)$ by the right regular representation, and $a \in \mathcal{A}$ acts on $F \in \operatorname{Ind}_{w}(V)$ by

$$
(\pi(a) F)(k)=\pi_{V}\left(\phi_{K}(k) a\right)(F(k)), \quad k \in K .
$$


The adjunction morphisms are given as follows. For a weak $(\mathcal{A}, K)$-module $V$, $\Phi_{V}: V \rightarrow \operatorname{Ind}_{w}($ For $V)$ is the matrix coefficient map, given by

$$
\Phi_{V}(v)(k)=\nu_{V}(k) v, \quad v \in V, k \in K .
$$

For an $\mathcal{A}$-module $W, \Psi_{W}: \operatorname{For}\left(\operatorname{Ind}_{w}(W)\right) \rightarrow W$ is the evaluation at 1.

The Zuckerman functor $\Gamma_{K, T}$ sends an $(\mathcal{A}, T)$-module $V$ into the invariants of $\operatorname{Ind}_{w}(V)$ with respect to the $(\mathfrak{k}, T)$-action $\lambda=\left(\lambda_{\mathfrak{k}}, \lambda_{T}\right)$, the tensor product of the left regular action of $(\mathfrak{k}, T)$ on $R(K)$ with the given action on $V$. To see that $\Gamma_{K, T}(V)$ is an $(\mathcal{A}, K)$-module, one can use the following formula relating the $\omega$-action of $\mathfrak{k}$ on the weak $(\mathcal{A}, K)$-module $\operatorname{Ind}_{w}(V)$ with the above described $\lambda$-action.

1.3.1. Lemma. For any $\xi \in \mathfrak{k}, F \in \operatorname{Ind}_{w}(V)$ and $k \in K$,

$$
(\omega(\xi) F)(k)=-\left(\lambda_{\mathfrak{k}}(\operatorname{Ad}(k) \xi) F\right)(k) .
$$

This formula was proved below 2.4 in [MP1]. One further shows

1.3.2. Theorem. The forgetful functor from $\mathcal{M}(\mathcal{A}, K)$ to $\mathcal{M}(\mathcal{A}, T)$ has a right adjoint, the Zuckerman functor $\Gamma_{K, T}$. For an $(\mathcal{A}, T)$-module $V$,

$$
\Gamma_{K, T}(V)=\operatorname{Hom}_{\mathfrak{k}, T}(\mathbb{C}, R(K, V)),
$$

where $\mathbb{C}$ is the trivial $(\mathfrak{k}, T)$-module, $R(K, V)$ is a $(\mathfrak{k}, T)$-module with respect to the action $\lambda=\left(\lambda_{\mathfrak{k}}, \lambda_{T}\right)$, and the $(\mathcal{A}, K)$-action on $\Gamma_{K, T}(V)$ comes from the $(\mathcal{A}, K)$ action on $R(K, V)$.

Being a right adjoint, $\Gamma_{K, T}$ is left exact, and it has right derived functors (since there are enough injectives). The following is a version of the Duflo-Vergne formula DV], which is an explicit formula for the derived Zuckerman functors holding under certain assumptions. It generalizes the formula for $\Gamma_{K, T}$ from 1.3.2. A proof can be found in [MP1, 1.6].

1.3.3. Theorem. Assume that $T$ is reductive and that $\mathcal{A}$ is a flat right $\mathcal{U}(\mathfrak{k})$-module for the right multiplication. Let $V$ be an $(\mathcal{A}, T)$-module. Then

$$
R^{p} \Gamma_{K, T}(V)=H^{p}(\mathfrak{k}, T ; R(K, V))
$$

for $p \in \mathbb{Z}_{+}$, where $R(K, V)$ is a $(\mathfrak{k}, T)$-module with respect to the action $\lambda$. The $(\mathcal{A}, K)$-action on $R^{p} \Gamma_{K, T}(V)$ comes from the $(\mathcal{A}, K)$-action on $R(K, V)$.

One can also consider the derived functor $R \Gamma_{K, T}$ between the corresponding derived categories. However, 1.3.3 does not generalize readily to a claim about this functor. The situation is better in the setting of equivariant derived categories which we define in the following.

\section{EquiVARIANT DERIVED CATEGORIES}

We are going to outline an algebraic construction of equivariant derived categories due to Beilinson and Ginzburg (see [BB, G] and [BL2]). There is also a geometric construction due to Bernstein and Lunts BL1]. 
2.1. Equivariant complexes. Let $(\mathcal{A}, K)$ be a Harish-Chandra pair. An equivariant $(\mathcal{A}, K)$-complex is a complex $V=V$ of weak $(\mathcal{A}, K)$-modules, together with a family of linear maps $i_{\xi}: V \rightarrow V$ of degree -1 , depending linearly on $\xi \in \mathfrak{k}$, and satisfying the following conditions:

$$
\begin{gathered}
i_{\xi} \pi(a)=\pi(a) i_{\xi}, \quad \xi \in \mathfrak{k}, a \in \mathcal{A} ; \\
i_{\operatorname{Ad}(k) \xi}=\nu(k) i_{\xi} \nu(k)^{-1}, \quad \xi \in \mathfrak{k}, k \in K ; \\
i_{\xi} i_{\eta}+i_{\eta} i_{\xi}=0, \quad \xi, \eta \in \mathfrak{k} \\
d i_{\xi}+i_{\xi} d=\omega(\xi), \quad \xi \in \mathfrak{k} .
\end{gathered}
$$

Here $\pi$ and $\nu$ are the actions of $\mathcal{A}$ and $K$ on $V, \omega=\nu-\pi$ is the action of $\mathfrak{k}$ from $\S 1.1$, and $d$ is the differential of the complex $V$.

Note that for any $\xi \in \mathfrak{k}, \omega(\xi)$ is a morphism of complexes of $\mathcal{A}$-modules, which is homotopic to zero, $i_{\xi}$ being the homotopy. It follows that $H^{p}(\omega(\xi))=0$ for any $p \in \mathbb{Z}$, so the cohomology modules of $V$ are $(\mathcal{A}, K)$-modules.

A morphism between two equivariant $(\mathcal{A}, K)$-complexes is a morphism of complexes of weak $(\mathcal{A}, K)$-modules which commutes with the $i_{\xi}$ 's. We denote the category of equivariant $(\mathcal{A}, K)$-complexes by $C(\mathcal{A}, K)$. It is clearly an abelian category.

2.2. DG algebras and modules. It will be convenient to reformulate the definition of equivariant complexes using the notion of DG modules over DG algebras. For more about these see [I]; see also [BL1] and [BL2]. The details needed here are written out in [P1]. The Lie algebra version of the theory is closely related to the theory of Lie superalgebras, which can be found in $\mathrm{Sch}$.

In the following, a complex will often be identified with the sum of its components with the natural grading. A differential graded (DG) Lie algebra is a complex of vector spaces $\mathfrak{d}$ with a graded bilinear operation $[-,-]$ called the supercommutator, satisfying the following conditions for any three homogeneous $X, Y, Z \in \mathfrak{d}$ :

$$
\begin{gathered}
{[X, Y]=-(-1)^{\operatorname{deg} X \operatorname{deg} Y}[Y, X] ;} \\
{[X,[Y, Z]]=[[X, Y], Z]+(-1)^{\operatorname{deg} X \operatorname{deg} Y}[Y,[X, Z]] ;} \\
d[X, Y]=[d X, Y]+(-1)^{\operatorname{deg} X}[X, d Y] .
\end{gathered}
$$

If we forget the differential and introduce the obvious $\mathbb{Z}_{2}$-grading, we get a Lie superalgebra.

A differential graded (DG) module over $\mathfrak{d}$ is a complex $V$ of vector spaces, with a graded action of $\mathfrak{d}$ on $V$ by linear endomorphisms, satisfying the properties

$$
[X, Y] v=X Y v-(-1)^{\operatorname{deg} X \operatorname{deg} Y} Y X v
$$

and

$$
d_{V}(X v)=\left(d_{\mathfrak{o}} X\right) v+(-1)^{\operatorname{deg} X} X d_{V} v
$$

for any (homogeneous) $X, Y \in \mathfrak{d}$ and $v \in V$.

An example of a DG Lie algebra is a Lie algebra considered as a complex concentrated in degree zero. The example important for us is the DG Lie algebra $\underline{\mathfrak{k}}$, corresponding to a Lie algebra $\mathfrak{k}$, defined as follows. As a complex of vector spaces, $\underline{\mathfrak{k}}$ is

$$
\ldots 0 \rightarrow \mathfrak{k} \stackrel{1}{\rightarrow} \mathfrak{k} \rightarrow 0 \ldots,
$$

with $\mathfrak{k}$ appearing in degrees -1 and 0 . The supercommutator is defined in the following way: on $\underline{\mathfrak{k}}^{0}=\mathfrak{k}$ it is just the commutator of $\mathfrak{k}, \underline{\mathfrak{k}}^{-1}$ supercommutes with 
itself, and for $X \in \underline{\mathfrak{k}}^{0}, \xi \in \underline{\mathfrak{k}}^{-1},[X, \xi]$ in $\underline{\mathfrak{k}}$ is the same as $[X, \xi]$ in $\mathfrak{k}$, understood as an element of $\underline{\mathfrak{k}}^{-1}$.

It is straightforward to check that in this way $\underline{\mathfrak{k}}$ becomes a DG Lie algebra. Furthermore, $K$ acts on $\underline{\mathfrak{k}}$ by adjoint action on each component. This is clearly an algebraic action by morphisms of complexes.

The reason for our interest in this is the following. Let $V$ be an equivariant $(\mathcal{A}, K)$-complex. We can define an action of $\underline{\mathfrak{k}}$ on $V$ as follows: $X \in \underline{\mathfrak{k}}^{0}=\mathfrak{k}$ acts by $\omega(X)$ while $\xi \in \underline{\mathfrak{k}}^{-1}$ acts by $i_{\xi}$. The fact that this is a DG action is equivalent to (EC3), (EC4), and the fact that $\omega$ is a representation of $\mathfrak{k}$. Furthermore, (EC1), (EC2), and the analogous properties of $\omega$ from 1.1, say that this is a $K$-equivariant action which commutes with the action of $\mathcal{A}$.

Now we want to pass to associative algebras. An associative DG algebra is a $\mathbb{Z}$-graded algebra $\mathcal{D}$ with unit, which is also a complex of vector spaces, such that for any homogeneous $x, y \in \mathcal{D}$,

$$
d(x y)=d(x) y+(-1)^{\operatorname{deg} x} x d(y) .
$$

Any (associative) DG algebra is a DG Lie algebra, if we define

$$
[a, b]=a b-(-1)^{\operatorname{deg} a \operatorname{deg} b} b a
$$

for homogeneous $a$ and $b$ in $\mathcal{D}$.

A morphism of two DG algebras is a map that is both an algebra homomorphism and a morphism of complexes. A (left) DG module over a DG algebra $\mathcal{D}$ is a graded (left) $\mathcal{D}$-module $M$, which is also a complex of vector spaces, such that the grading of the module is the same as the grading of the complex, and the following condition holds:

$$
d_{M}(x m)=\left(d_{\mathcal{D}} x\right) m+(-1)^{\operatorname{deg} x} x\left(d_{M} m\right)
$$

for any homogeneous $x \in \mathcal{D}$ and $m \in M$.

An example of a DG algebra is any associative algebra considered as a complex concentrated in degree 0 . The most important examples for our purposes are the universal enveloping algebras of DG Lie algebras (concretely, of $\underline{\mathfrak{k}}$ ). These are defined analogously to ordinary enveloping algebras, and enveloping algebras of superalgebras (see $[\mathrm{Sch})$. Starting with a DG Lie algebra $\mathfrak{d}$, we first define its tensor algebra $T(\mathfrak{d})$; as an algebra, it is the ususal tensor algebra. The grading is given by

$$
\operatorname{deg}\left(X_{1} \otimes \cdots \otimes X_{k}\right)=\operatorname{deg} X_{1}+\cdots+\operatorname{deg} X_{k}
$$

for homogeneous $X_{1}, \ldots, X_{k} \in \mathfrak{d}$, and the differential is given by

$d\left(X_{1} \otimes \cdots \otimes X_{k}\right)=\sum_{i=1}^{k}(-1)^{\operatorname{deg} X_{1}+\ldots \operatorname{deg} X_{i-1}} X_{1} \otimes \cdots \otimes X_{i-1} \otimes d\left(X_{i}\right) \otimes X_{i+1} \otimes \cdots \otimes X_{k}$

and $d(1)=0$. It is easy to check that in this way $T(\mathfrak{d})$ becomes a DG algebra. Then we consider the two-sided ideal $\mathcal{I}$ of $T(\mathfrak{g})$ generated by the elements of the form

$$
X \otimes Y-(-1)^{\operatorname{deg} X \operatorname{deg} Y} Y \otimes X-[X, Y]
$$

for homogeneous $X, Y \in \mathfrak{d}$. One checks that $\mathcal{I}$ is a DG ideal, i.e., a graded ideal closed under the differential. It follows that the universal enveloping algebra

$$
\mathcal{U}(\mathfrak{d})=T(\mathfrak{d}) / \mathcal{I}
$$


of $\mathfrak{d}$ is a DG algebra. There is an obvious DG Lie algebra morphism $\phi: \mathfrak{d} \rightarrow \mathcal{U}(\mathfrak{d})$, and the following universal property holds: For any DG algebra $\mathcal{D}$ and a DG Lie algebra morphism $f$ from $\mathfrak{d}$ into $\mathcal{D}$ (considered as a DG Lie algebra as above), there is a unique DG algebra morphism $\tilde{f}$ from $\mathcal{U}(\mathfrak{d})$ into $\mathcal{D}$ such that $f=\tilde{f} \circ \phi$. In particular, $M$ is a DG module over $\mathfrak{d}$ if and only if it is a DG module over $\mathcal{U}(\mathfrak{d})$.

The proof is the same as for Lie algebras, as is the proof of the following version of the Poincaré-Birkhoff-Witt Theorem.

2.2.1. Proposition. Let $X_{1}, \ldots, X_{n}, Y_{1}, \ldots, Y_{m}$ be a basis of $\mathfrak{d}$ consisting of homogeneous elements, with $X_{k}$ 's even and $Y_{k}$ 's odd. Then

$$
\left(X_{1}^{i_{1}} \ldots X_{n}^{i_{n}} Y_{1}^{j_{1}} \ldots Y_{m}^{j_{m}}\right)_{i_{k} \in \mathbb{Z}_{+}, j_{k} \in\{0,1\}}
$$

is a basis of $\mathcal{U}(\mathfrak{d})$.

Using this and a straightforward calculation of the differential, we obtain the following (known) result which goes back to Cartier; see BL2, 1.9.7. Recall that the standard complex $N(\mathfrak{k})$ of a Lie algebra $\mathfrak{k}$ is defined by

$$
N^{p}(\mathfrak{k})=\mathcal{U}(\mathfrak{k}) \otimes \bigwedge^{-p}(\mathfrak{k}),
$$

with the differential given by

$$
\begin{aligned}
d\left(u \otimes \lambda_{1} \wedge \cdots \wedge \lambda_{p}\right) & =\sum_{i=1}^{p}(-1)^{i-1} u \lambda_{i} \otimes \lambda_{1} \wedge \cdots \wedge \hat{\lambda_{i}} \wedge \cdots \wedge \lambda_{p} \\
& +\sum_{j<i}(-1)^{j+i} u \otimes\left[\lambda_{j}, \lambda_{i}\right] \wedge \lambda_{1} \wedge \cdots \wedge \hat{\lambda_{j}} \wedge \cdots \wedge \hat{\lambda_{i}} \wedge \cdots \wedge \lambda_{p} .
\end{aligned}
$$

2.2.2. Proposition. Let $\mathfrak{k}$ be a Lie algebra. As a complex of vector spaces, $\mathcal{U}(\underline{\mathfrak{k}})$ is isomorphic to the standard complex $N(\mathfrak{k})$ of $\mathfrak{k}$.

In particular, it follows that $N(\mathfrak{k})$ has the structure of a DG algebra. The same structure is described in [MP1, §3].

We see that our equivariant $(\mathcal{A}, K)$-complexes are DG modules over $N(\mathfrak{k})$. This suggests the following definitions. Let $\mathcal{D}$ be a DG algebra with an algebraic action $\chi$ of $K$ by (DG) automorphisms, and with a morphism $\rho: \mathfrak{k} \rightarrow \mathcal{D}$ of DG Lie algebras, such that the same conditions as for $\mathcal{A}$ are satisfied: $\rho$ is $K$-equivariant, and the differential of the action $\chi$ is the same as the action of $\mathfrak{k}$ on $\mathcal{D}$ given by inner derivations $[\rho(\xi),-]$. Such a $\mathcal{D}$ is called a Harish-Chandra DG algebra in BL2. Note that $\rho$ being a morphism of DG Lie algebras implies that its image is contained in the cycles of $\mathcal{D}^{0}$.

The main example for $\mathcal{D}$ is the standard complex of $\mathfrak{k}$, or of a Lie algebra $\mathfrak{g}$ such that $(\mathfrak{g}, K)$ is a Harish-Chandra pair. Other examples are $\mathcal{D}=\mathbb{C}$ or $\mathcal{D}=\mathcal{U}(\mathfrak{k})$ (concentrated in degree 0 ). In these examples $K$ acts trivially on $\mathbb{C}$ and by the adjoint action on $\mathcal{U}(\mathfrak{k}), N(\mathfrak{k})$ and $N(\mathfrak{g})$, while $\rho$ is the zero map, respectively the natural inclusion.

We now define an $(\mathcal{A}, K, \mathcal{D})$-module to be a complex $V$ of vector spaces, with actions $\pi$ of $\mathcal{A}, \nu$ of $K$ and $\omega$ of $\mathcal{D}$, such that the following conditions hold:

(AKD1) $\mathcal{A}$ and $K$ act by morphisms of complexes, the $K$-action is algebraic, and the $\mathcal{D}$-action is a DG-action;

(AKD2) $\pi$ and $\omega$ are both $K$-equivariant and they commute;

(AKD3) The representations $\pi, \omega$ and $\nu$ of $\mathfrak{k}$ satisfy the relation $\pi+\omega=\nu$. 
A morphism between two $(\mathcal{A}, K, \mathcal{D})$-modules is a morphism of complexes which preserves all the actions. The category $\mathcal{M}(\mathcal{A}, K, \mathcal{D})$ of all $(\mathcal{A}, K, \mathcal{D})$-modules is clearly abelian. Examples of interest are the following:

(1) $\mathcal{D}=N(\mathfrak{k})$; then $\mathcal{M}(\mathcal{A}, K, \mathcal{D})$ is the category of equivariant $(\mathcal{A}, K)$-complexes.

(2) $\mathcal{D}=\mathcal{U}(\mathfrak{k})$; then $\mathcal{M}(\mathcal{A}, K, \mathcal{D})$ is the category of complexes of weak $(\mathcal{A}, K)$ modules.

(3) $\mathcal{D}=\mathbb{C}$; then $\mathcal{M}(\mathcal{A}, K, \mathcal{D})$ is the category of complexes of $(\mathcal{A}, K)$-modules.

We will describe the relationship between these categories in 2.5.2.

Let us now briefly discuss right DG modules. A right module $M$ over $\mathcal{D}$ is a right DG module if

$$
d_{M}(m x)=\left(d_{M} m\right) x+(-1)^{\operatorname{deg} m} m\left(d_{\mathcal{D}} x\right)
$$

for any homogeneous $x \in \mathcal{D}$ and $m \in M$.

We can also define the opposite DG algebra $\mathcal{D}^{o p p}$ : As a complex of vector spaces it is the same as $\mathcal{D}$, and the multiplication is given by

$$
x \circ y=(-1)^{\operatorname{deg} x \operatorname{deg} y} y x
$$

for homogeneous $x$ and $y$, where $y x$ is the multiplication in $\mathcal{D}$.

Then the category of left (respectively right) DG modules over $\mathcal{D}$ is naturally isomorphic to the category of right (respectively left) DG modules over $\mathcal{D}^{o p p}$. Namely, if $M$ is a left DG module over $\mathcal{D}$, then it becomes a right DG module over $\mathcal{D}^{o p p}$ if we define

$$
m \circ x=(-1)^{\operatorname{deg} x \operatorname{deg} m} x m
$$

for homogeneous $x \in \mathcal{D}$ and $m \in M$.

In case $\mathcal{D}=\mathcal{U}(\mathfrak{d})$ for a DG Lie algebra $\mathfrak{d}, \mathcal{D}^{o p p}$ can be identified with $\mathcal{D}$. Namely, let us define $\iota: \mathfrak{d} \rightarrow \mathcal{U}(\mathfrak{d})^{o p p}$ by

$$
\iota(X)={ }^{\iota} X=-X, \quad X \in \mathfrak{d} .
$$

It is easily checked to be a morphism of DG Lie algebras. Therefore it defines a DG algebra morphism from $\mathcal{U}(\mathfrak{d})$ into $\mathcal{U}(\mathfrak{d})^{o p p}$. In a Poincaré-Birkhoff-Witt basis, $\iota$ acts on monomials by flipping the order and putting an appropriate sign. So it is clearly an isomorphism. We will call $\iota$, understood as a map from $\mathcal{U}(\mathfrak{d})$ into itself, the principal anti-automorphism of $\mathcal{U}(\mathfrak{d})$. It is a generalization of the principal anti-automorphism in the case of ordinary Lie algebras. It clearly satisfies

$$
{ }^{\iota}(u v)=(-1)^{\operatorname{deg} u \operatorname{deg} v \quad \iota} v^{\iota} u
$$

for homogeneous $u, v \in \mathcal{U}(\mathfrak{d})$.

Note that we can use $\iota$ to pass from left to right DG modules over $\mathcal{U}(\mathfrak{d})$ and vice versa. Indeed, the following lemma holds.

2.2.3. Lemma. If $\omega$ is a left (respectively right) action of $\mathcal{U}(\mathfrak{d})$ on $M$, then setting

$$
\tilde{\omega}(u)(m)=(-1)^{\operatorname{deg} u \operatorname{deg} m} \omega\left({ }^{\iota} u\right)(m)
$$

for homogeneous $u \in \mathcal{U}(\mathfrak{d})$ and $m \in M$ defines a right (respectively left) action of $\mathcal{U}(\mathfrak{d})$ on $M$.

The above principal anti-automorphism is actually the antipode map for a Hopf algebra structure on $\mathcal{U}(\mathfrak{d})$. Let us briefly describe this structure.

Let us first remark that for DG algebras $\mathcal{D}$ and $\mathcal{E}$, we can define their tensor product $\mathcal{D} \bar{\otimes} \mathcal{E}$. It is equal to $\mathcal{D} \otimes \mathcal{E}$ as a vector space, it has the standard tensor 
product grading and differential (see the definition of the tensor algebra above), and the multiplication is given by

$$
(x \otimes y)\left(x^{\prime} \otimes y^{\prime}\right)=(-1)^{\operatorname{deg} y \operatorname{deg} x^{\prime}}\left(x x^{\prime} \otimes y y^{\prime}\right)
$$

for $x, x^{\prime} \in \mathcal{D}$ and $y, y^{\prime} \in \mathcal{E}$. It is straightforward to check that $\mathcal{D} \bar{\otimes} \mathcal{E}$ is a DG algebra.

We now define $c: \mathfrak{d} \rightarrow \mathcal{U}(\mathfrak{d}) \bar{\otimes} \mathcal{U}(\mathfrak{d})$ by

$$
c(X)=X \otimes 1+1 \otimes X, \quad X \in \mathfrak{d} .
$$

One easily checks that $c$ is a morphism of DG Lie algebras, hence it extends in a unique way to a DG morphism $c: \mathcal{U}(\mathfrak{d}) \rightarrow \mathcal{U}(\mathfrak{d}) \bar{\otimes} \mathcal{U}(\mathfrak{d})$. This is the coproduct for $\mathcal{U}(\mathfrak{d})$.

The counit $\varepsilon: \mathcal{U}(\mathfrak{d}) \rightarrow \mathbb{C}$ is defined by the requirements $\varepsilon(\mathfrak{d} \mathcal{U}(\mathfrak{d}))=0$ and $\varepsilon(1)=1$.

It is now straightforward to check the following:

2.2.4. Proposition. With the above definitions, $\mathcal{U}(\mathfrak{d})$ becomes a Hopf algebra. Moreover, the Hopf algebra structure is compatible with the DG algebra structure, in the sense that $c$ and $\varepsilon$ are not just algebra morphisms, but DG algebra morphisms.

2.3. Homotopic and derived categories of $(\mathcal{A}, K, \mathcal{D})$-modules. We first define the translation functor for $(\mathcal{A}, K, \mathcal{D})$-modules. Let $V$ be an $(\mathcal{A}, K, \mathcal{D})$-module. Then $T(V)=V[1]$ is the same as $V$ as a weak $(\mathcal{A}, K)$-module. The grading is shifted: $T(V)^{i}=V^{i+1}$, the differential is given by $d_{T(V)}=-d_{V}$, and the $\mathcal{D}$-action is twisted:

$$
\omega_{T(V)}(x) v=(-1)^{\operatorname{deg} x} \omega_{V}(x) v,
$$

for homogeneous $x \in \mathcal{D}$ and $v \in V$. It is clear that $T(V)$ is again an $(\mathcal{A}, K, \mathcal{D})$ module. If we define the action of $T$ on morphisms just by shifting the degree: $T(f)^{i}=f^{i+1}$, then $T$ clearly becomes a functor. Moreover, $T$ is an autoequivalence of the category $\mathcal{M}(\mathcal{A}, K, \mathcal{D})$; the inverse is given by similar shifting but in the opposite direction.

We say that two $(\mathcal{A}, K, \mathcal{D})$ morphisms $f$ and $g$ from $V$ to $W$ are homotopic if there exists $h: V \rightarrow T^{-1}(W)$, which is an $(\mathcal{A}, K)$-morphism and a $\mathcal{D}$-morphism of degree 0 , but not necessarily a morphism of complexes (so it is not a morphism in $\mathcal{M}(\mathcal{A}, K, \mathcal{D}))$, such that

$$
f-g=h d_{V}+d_{W} h .
$$

For equivariant complexes $(\mathcal{D}=N(\mathfrak{k}))$, this condition just means that $f$ and $g$ are homotopic as morphisms of complexes of weak $(\mathcal{A}, K)$-modules, via a homotopy which anticommutes with $i_{\xi}$ 's.

We now define the homotopic category $K(\mathcal{A}, K, \mathcal{D})$. Its objects are $(\mathcal{A}, K, \mathcal{D})$ modules, and its morphisms are homotopy classes of morphisms in $\mathcal{M}(\mathcal{A}, K, \mathcal{D})$.

2.3.1. Proposition. The category $K(\mathcal{A}, K, \mathcal{D})$ is a triangulated category.

Proof. The proof is basically the same as for the homotopic category of complexes over an abelian category. First, it is clear that the above translation functor descends to $K(\mathcal{A}, K, \mathcal{D})$; namely, if $h$ is a homotopy from $f$ to 0 , then $-h$ shifted in degree by 1 is a homotopy from $T(f)$ to 0 .

Next we define the cone of a morphism. Let $f: V \rightarrow W$ be a morphism in $\mathcal{M}(\mathcal{A}, K, \mathcal{D})$. As a weak $(\mathcal{A}, K)$-module and a graded $\mathcal{D}$-module, the cone of $f$ is 
$C_{f}=T(V) \oplus W$. The differential of $C_{f}$ is

$$
d_{f}=\left(\begin{array}{cc}
d_{T(V)} & 0 \\
T(f) & d_{W}
\end{array}\right) .
$$

Then one easily checks that $C_{f}$ is an $(\mathcal{A}, K, \mathcal{D})$-module.

The inclusion $i_{f}: W \rightarrow C_{f}$ and the projection $p_{f}: C_{f} \rightarrow T(V)$ are clearly $(\mathcal{A}, K, \mathcal{D})$-morphisms. In this way we obtain the standard triangles

$$
V \stackrel{f}{\rightarrow} W \stackrel{i_{f}}{\rightarrow} C_{f} \stackrel{p_{f}}{\rightarrow} T(V)
$$

We should show that these triangles are well-defined for a morphism $f$ in the homotopic category $K(\mathcal{A}, K, \mathcal{D})$, i.e., that homotopic $f$ and $g$ give isomorphic triangles (in the obvious sense). Then we can define the distinguished triangles as all triangles in $K(\mathcal{A}, K, \mathcal{D})$ which are isomorphic (in $K(\mathcal{A}, K, \mathcal{D})$ ) to some standard triangle. Finally, we should check the axioms of triangulated categories.

Note, however, that both our homotopies and cones are also homotopies and cones in the category of complexes of weak $(\mathcal{A}, K)$-modules. They only have the additional structure of graded $\mathcal{D}$-morphisms, respectively modules. If we now go through the classical proof of the fact that $K\left(\mathcal{M}(\mathcal{A}, K)_{w}\right)$ is a triangulated category, we see that for the proof we only need to construct certain morphisms and homotopies. All these are given by matrices and all the components of these matrices are clearly graded $\mathcal{D}$-morphisms in our case. Therefore the classical proof goes through without changes.

To obtain the derived category $D(\mathcal{A}, K, \mathcal{D})$ of $(\mathcal{A}, K, \mathcal{D})$-modules, we localize $K(\mathcal{A}, K, \mathcal{D})$ with respect to quasi-isomorphisms, that is, morphisms in $K(\mathcal{A}, K, \mathcal{D})$ which induce isomorphisms on cohomology.

2.3.2. Lemma. Quasi-isomorphisms in $K(\mathcal{A}, K, \mathcal{D})$ form a saturated localizing class (or multiplicative system) compatible with triangulation.

Proof. The proof of this lemma is analogous to the standard proof of the same fact for the homotopic category of an abelian category. The simplest approach is to first note that taking $p^{\text {th }}$ cohomology is a cohomological functor for every $p$, i.e., that for any distinguished triangle

$$
V \stackrel{f}{\rightarrow} W \rightarrow Z \rightarrow T(V)
$$

in $K(\mathcal{A}, K, \mathcal{D})$ we have a corresponding long exact sequence of vector spaces

$$
\cdots \rightarrow H^{p-1}(Z) \rightarrow H^{p}(V) \rightarrow H^{p}(W) \rightarrow H^{p}(Z) \rightarrow H^{p+1}(V) \cdots .
$$

This immediately implies that $f$ is a quasi-isomorphism if and only if $Z$ is acyclic, i.e., $H^{p}(Z)=0$ for all $p$.

The above claim about quasi-isomorphisms is therefore equivalent to the fact that acyclic objects form a null system in $K(\mathcal{A}, K, \mathcal{D})$ (as defined in [KS], Definition 1.6.6), with the additional condition corresponding to the saturation condition: any direct summand of an object in $\mathcal{N}$ is in $\mathcal{N}$. It is trivial to check that this is indeed the case. For details, see [P1], 5.2.2 and 1.1.9.

It is a standard fact (see [KS], Proposition 1.6.9, or [GM]) that if the above conditions are satisfied, then the localized category $D(\mathcal{A}, K, \mathcal{D})$ inherits the structure of a triangulated category. The distinguished triangles in $D(\mathcal{A}, K, \mathcal{D})$ are simply all triangles isomorphic in $D(\mathcal{A}, K, \mathcal{D})$ to distinguished triangles in $K(\mathcal{A}, K, \mathcal{D})$. 
2.3.3. Corollary. The category $D(\mathcal{A}, K, \mathcal{D})$ is a triangulated category.

In case $\mathcal{D}=N(\mathfrak{k})$, which is our main example, the category $D(\mathcal{A}, K, N(\mathfrak{k}))$ is called the equivariant derived category of $(\mathcal{A}, K)$-modules. We will sometimes denote it just by $D(\mathcal{A}, K)$, and the corresponding homotopic category by $K(\mathcal{A}, K)$.

All the above goes through without changes for complexes bounded above, below, or from both sides. The corresponding derived categories are denoted by $D^{*}(\mathcal{A}, K, \mathcal{D})$, where $*$ can be,-+ , or $b$ (or nothing in the case of the full derived category as above).

Another important property of equivariant derived categories is the fact that we can embed the category $\mathcal{M}(\mathcal{A}, K)$ into $D^{b}(\mathcal{A}, K)$, via the functor $D$ sending a module $M$ into the equivariant complex concentrated in degree 0 and having $M$ as its zeroth component. The functor $D$ is fully faithful, and its image is a generating class of $D^{b}(\mathcal{A}, K)$. This is completely analogous to the embedding of an abelian category $\mathcal{C}$ into its derived category $D^{b}(\mathcal{C})$. The proof in our present situation is also completely analogous. It is based on the notion of truncations, which are defined in a usual way, and have analogous properties as their classical counterparts.

2.4. Functors between homotopic categories. In the next sections we will construct various functors on the level of $(\mathcal{A}, K, \mathcal{D})$-modules, and then we will need to show that they make sense on the level of homotopic categories. In this section we give a criterion that will be useful in such situations.

Let us fix two triples, $(\mathcal{A}, K, \mathcal{D})$ and $(\mathcal{B}, T, \mathcal{E})$, as in Section 2.2. Let $F_{1}$ be an additive functor from $\mathcal{M}(\mathcal{A}, K, \mathcal{D})$ into $\mathcal{M}(\mathcal{B}, T, \mathcal{E})$, and let $F_{2}$ be an additive functor between the corresponding categories of graded modules, $\mathcal{M}^{G R}(\mathcal{A}, K, \mathcal{D})$ and $\mathcal{M}^{G R}(\mathcal{B}, K, \mathcal{E})$. Here morphisms of graded modules are required to be of degree 0 . Assume that the diagram

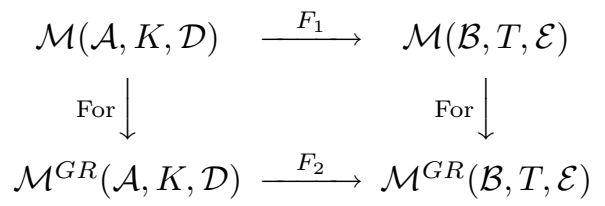

commutes. Here For denotes the obvious forgetful functors. Then we will loosely denote both $F_{1}$ and $F_{2}$ by $F$ and think of $F$ as a functor defined both on the level of DG modules and on the level of graded modules, in a compatible way. We also assume that $F$ commutes with translations, i.e., that both $F_{1}$ and $F_{2}$ commute with translations.

Now we need to define the Hom -complex. Let $V$ and $W$ be two objects of $\mathcal{M}(\mathcal{A}, K, \mathcal{D})$. First we define $\operatorname{Hom}_{\mathbb{C}}(V, W)$ to be just the standard Hom of complexes. In other words,

$$
\operatorname{Hom}_{\mathbb{C}}^{k}(V, W)=\prod_{j} \operatorname{Hom}_{\mathbb{C}}\left(V^{j}, W^{j+k}\right)
$$

and the differential is given by $d f=d_{W} \circ f-(-1)^{\operatorname{deg} f} f \circ d_{V}$ for a homogeneous $f \in \operatorname{Hom}_{\mathbb{C}}(V, W)$. More precisely,

$$
d^{k} f=\left(d_{W}^{j+k} \circ f_{j}-(-1)^{k} f_{j+1} \circ d_{V}^{j}\right),
$$

for $f=\left(f_{j}\right) \in \prod_{j} \operatorname{Hom}_{\mathbb{C}}\left(V^{j}, W^{j+k}\right)$. 
Now we define $\operatorname{Hom}_{(\mathcal{A}, K, \mathcal{D})}(V, W)$ as the linear subspace of $\operatorname{Hom}_{\mathbb{C}}(V, W)$, consisting of $(\mathcal{A}, K)$-morphisms whose homogeneous components $f$ satisfy

$$
f(x v)=(-1)^{\operatorname{deg} f \operatorname{deg} x} x f(v)
$$

for any homogeneous $x \in \mathcal{D}$ and $v \in V$. It is easy to see that $\operatorname{Hom}_{(\mathcal{A}, K, \mathcal{D})}(V, W)$ is actually a subcomplex of $\operatorname{Hom}_{\mathbb{C}}(V, W)$, and that $\operatorname{Hom}_{(\mathcal{A}, K, \mathcal{D})}$ is an additive bifunctor into the category $C\left(V e c t_{\mathbb{C}}\right)$ of complexes of vector spaces.

Note that $\operatorname{Hom}_{\mathcal{M}(\mathcal{A}, K, \mathcal{D})}(V, W)$ consists of 0 -cycles in $\operatorname{Hom}_{(\mathcal{A}, K, \mathcal{D})}(V, W)$, and that $\operatorname{Hom}_{K(\mathcal{A}, K, \mathcal{D})}(V, W)$ is the zeroth cohomology space of the complex $\operatorname{Hom}_{(\mathcal{A}, K, \mathcal{D})}(V, W)$.

Now we return to a functor $F$ as above. Since $(\mathcal{A}, K, \mathcal{D})$-maps of degree $k$ between $V$ and $W$ are the same as $(\mathcal{A}, K, \mathcal{D})$-maps of degree 0 between $V$ and $W[k]$, and $F$ commutes with translations, we see that $F$ defines a linear map

$$
F=F_{V, W}: \operatorname{Hom}_{(\mathcal{A}, K, \mathcal{D})}(V, W) \rightarrow \operatorname{Hom}_{(\mathcal{B}, T, \mathcal{E})}(F(V), F(W)) .
$$

2.4.1. Theorem. Let $F$ be a functor as above, such that for any $V$ and $W$ in $\mathcal{M}(\mathcal{A}, K, \mathcal{D})$ the linear map $F_{V, W}$ defined above is a morphism of complexes. Then $F$ defines an exact functor from $K(\mathcal{A}, K, \mathcal{D})$ into $K(\mathcal{B}, T, \mathcal{E})$.

Remark. Recall that an exact functor between triangulated categories is a functor commuting with translation and sending distinguished triangles to distinguished triangles.

Proof. Since $F_{V, W}$ is a morphism of complexes, it defines a map on the level of cohomology, so it defines a map on the level of homotopic classes of morphisms. Therefore, to finish the proof, it is enough to show that $F$ preserves cones (we already assumed that $F$ commutes with translation).

Let $f: V \rightarrow W$ be a morphism in $\mathcal{M}(\mathcal{A}, K, \mathcal{D})$. Since $F$ is additive and commutes with translation, $F\left(C_{f}\right)=C_{F(f)}$ as graded $(\mathcal{B}, K, \mathcal{E})$-modules. So we only need to show that the differential of $F\left(C_{f}\right)$ is equal to the differential $d_{F(f)}$ of the cone $C_{F(f)}$, i.e., that

$$
d_{F\left(C_{f}\right)}=\left(\begin{array}{cc}
d_{T(F(V))} & 0 \\
T(F(f)) & d_{F(W)}
\end{array}\right) .
$$

This is proved by a straightforward calculation of the matrix coefficients of $d_{F\left(C_{f}\right)}$.

Let us remark that in the classical situation one usually starts with a functor on the level of abelian categories, and then lets it act on complexes by acting on all components and on differentials. The action on morphisms of complexes is given componentwise. Such a functor obviously acts on graded objects and morphisms, and it trivially meets the conditions of 2.4.1. Therefore such a functor always defines a functor on the level of homotopic categories.

The first functor for which the above conditions are nontrivial is $\operatorname{Hom}_{(\mathcal{A}, K, \mathcal{D})}$ itself. Let $V$ be an $(\mathcal{A}, K, \mathcal{D})$-module. Then $\operatorname{Hom}_{(\mathcal{A}, K, \mathcal{D})}(V,-)$ is a functor from $\mathcal{M}(\mathcal{A}, K, \mathcal{D})$ into $C\left(V_{e c t}\right)$, which is compatibly defined on the level of graded modules; in both cases it is defined on morphisms by $\alpha \mapsto \alpha \circ-$.

It is easy to show that $\operatorname{Hom}_{(\mathcal{A}, K, \mathcal{D})}(V,-)$ commutes with translation. It remains to show that the map sending $\alpha \in \operatorname{Hom}_{(\mathcal{A}, K, \mathcal{D})}\left(W_{1}, W_{2}\right)$ into

$$
\tilde{\alpha}=\alpha \circ-\in \operatorname{Hom}_{\mathbb{C}}\left(\operatorname{Hom}_{(\mathcal{A}, K, \mathcal{D})}\left(V, W_{1}\right), \operatorname{Hom}_{(\mathcal{A}, K, \mathcal{D})}\left(V, W_{2}\right)\right)
$$


is a morphism of complexes. Since $\operatorname{Hom}_{(\mathcal{A}, K, \mathcal{D})}(V, W)$ is a subcomplex of $\operatorname{Hom}_{\mathbb{C}}(V, W)$, this follows immediately from the first part of the following lemma.

2.4.2. Lemma. Let $V, W_{1}$ and $W_{2}$ be complexes of vector spaces.

(i) For $\alpha \in \operatorname{Hom}_{\mathbb{C}}\left(W_{1}, W_{2}\right)$, define $\tilde{\alpha} \in \operatorname{Hom}_{\mathbb{C}}\left(\operatorname{Hom}_{\mathbb{C}}\left(V, W_{1}\right), \operatorname{Hom}_{\mathbb{C}}\left(V, W_{2}\right)\right)$ by $\tilde{\alpha}=\alpha \circ-$. Then the map $\alpha \mapsto \tilde{\alpha}$ is a morphism of complexes.

(ii) The map $\alpha \mapsto \alpha \otimes i d$ from $\operatorname{Hom}_{\mathbb{C}}\left(W_{1}, W_{2}\right)$ into $\operatorname{Hom}_{\mathbb{C}}\left(W_{1} \otimes_{\mathbb{C}} V, W_{2} \otimes_{\mathbb{C}} V\right)$ is a morphism of complexes.

The proof of the lemma is straightforward, and hence we get

2.4.3. Proposition. For any $(\mathcal{A}, K, \mathcal{D})$-module $V$, $\operatorname{Hom}_{(\mathcal{A}, K, \mathcal{D})}(V,-)$ defines an exact functor from $K(\mathcal{A}, K, \mathcal{D})$ into $K\left(V e c t_{\mathbb{C}}\right)$.

We remark that similarly one can prove the analogous claim for the first variable, and it is then easy to show that $\operatorname{Hom}_{(\mathcal{A}, K, \mathcal{D})}$ is an exact bifunctor from $K(\mathcal{A}, K, \mathcal{D})^{o p p} \times K(\mathcal{A}, K, \mathcal{D})$ into $K\left(\right.$ Vect $\left._{\mathbb{C}}\right)$.

The last question we want to address in this section is adjunction of functors on the homotopic level. The following easy fact is enough for most purposes.

2.4.4. Proposition. Let $F: \mathcal{M}(\mathcal{A}, K, \mathcal{D}) \rightarrow \mathcal{M}(\mathcal{B}, T, \mathcal{E})$ be left adjoint to the functor $G: \mathcal{M}(\mathcal{B}, T, \mathcal{E}) \rightarrow \mathcal{M}(\mathcal{A}, K, \mathcal{D})$. Suppose that both functors define the same named functors on the level of homotopic categories. Then $F$ and $G$ are adjoint functors between the homotopic categories.

Proof. The adjunction morphisms on the level of homotopic categories are defined as the homotopy classes of the adjunction morphisms on the level of complexes. The compositions in the definition of adjunction are the identities because it is so on the level of representatives.

In most situations the reason for $F$ and $G$ to define functors on the level of homotopic categories will be the fact that they satisfy the conditions of 2.4.1. If this is the case, and if the adjunction morphisms $\Phi_{X}$ and $\Psi_{Y}$ are natural not only with respect to morphisms (which are in particular morphisms of complexes), but also with respect to graded morphisms (which are not necessarily morphisms of complexes), then we actually have the following stronger version of 2.4 .4 (2.4.4 follows from it by taking zeroth cohomology):

2.4.5. Theorem. Let $F: \mathcal{M}(\mathcal{A}, K, \mathcal{D}) \rightarrow \mathcal{M}(\mathcal{B}, T, \mathcal{E})$ be left adjoint to $G:$ $\mathcal{M}(\mathcal{B}, T, \mathcal{E}) \rightarrow \mathcal{M}(\mathcal{A}, K, \mathcal{D})$. Assume that both functors satisfy the conditions of 2.4.1, and that the adjunction natural transformations $\Phi$ and $\Psi$ are natural with respect to graded morphisms. Then

$$
\operatorname{Hom}_{(\mathcal{B}, T, \mathcal{E})}(F X, Y) \cong \operatorname{Hom}_{(\mathcal{A}, K, \mathcal{D})}(X, G Y)
$$

for any $X \in \mathcal{M}(\mathcal{A}, K, \mathcal{D})$ and $Y \in \mathcal{M}(\mathcal{B}, T, \mathcal{E})$.

Proof. The proof is similar to the standard proof of the fact that adjunction of two functors (defined by the equality $\operatorname{Hom}(F X, Y)=\operatorname{Hom}(X, G Y)$ ) is equivalent to the existence of adjunction natural transformations. Namely, one defines

$$
\alpha_{X, Y}: \operatorname{Hom}_{(\mathcal{B}, T, \mathcal{E})}(F X, Y) \rightarrow \operatorname{Hom}_{(\mathcal{A}, K, \mathcal{D})}(X, G Y)
$$

and $\beta_{X, Y}$ in the opposite direction by

$$
\alpha_{X, Y}(f)=G(f) \circ \Phi_{X} ; \quad \beta_{X, Y}(g)=\Psi_{Y} \circ F(g) .
$$


This makes sense since $F$ and $G$ are defined on graded morphisms. The proof that $\alpha$ and $\beta$ are natural, and that their compositions in both orders give identity morphisms, is the same as in the classical proof (but using our present assumptions). The only thing we have to show is that $\alpha_{X, Y}$ is a morphism of complexes. This follows from the fact that $G$ induces a morphism of complexes between Hom -spaces, and that $\Phi_{X}$ is a morphism of complexes.

2.5. Change of DG algebras. Let $(\mathcal{A}, K, \mathcal{D})$ and $(\mathcal{A}, K, \mathcal{E})$ be triples as in Section 2.2 , with $\phi, \psi, \chi_{\mathcal{E}}, \chi_{\mathcal{D}}, \rho_{\mathcal{E}}$ and $\rho_{\mathcal{D}}$ the corresponding maps. Let $\alpha: \mathcal{D} \rightarrow \mathcal{E}$ be a morphism of DG algebras, intertwining the $K$-actions, and such that

$$
\rho_{\mathcal{E}}=\alpha \circ \rho_{\mathcal{D}} .
$$

Then $\alpha$ defines a forgetful functor For from $\mathcal{M}(\mathcal{A}, K, \mathcal{E})$ into $\mathcal{M}(\mathcal{A}, K, \mathcal{D})$ in the obvious way: The $\mathcal{D}$ action is obtained by composing the $\mathcal{E}$-action with $\alpha$. We want to construct adjoints of For.

For simplicity, we assume that both $\mathcal{D}$ and $\mathcal{E}$ have principal anti-automorphisms, commuting with the $K$-action, which we denote by $\iota$ in each case; we also assume that ${ }^{\iota} \rho(\xi)=-\rho(\xi)$ for any $\xi \in \mathfrak{k}$ and that the morphism $\alpha$ intertwines the $\iota$ 's. This will be satisfied in all the examples we need, and it simplifies matters by allowing us to work with left modules only.

To construct the left adjoint, let $V$ be an $(\mathcal{A}, K, \mathcal{D})$-module, with actions $\pi_{V}$ of $\mathcal{A}, \nu_{V}$ of $K$ and $\omega_{V}$ of $\mathcal{D}$. We consider it a right DG module over $\mathcal{D}$ as in 2.2.3. Now we consider $V \otimes_{\mathcal{D}} \mathcal{E}$, with $\mathcal{D}$-action on $\mathcal{E}$ being the left multiplication. This is a right DG module over $\mathcal{E}$, for the right multiplication in the second factor. We turn it into a left $\mathcal{E}$-module using 2.2 .3 again. In other words, the $\mathcal{E}$-action on $V \otimes_{\mathcal{D}} \mathcal{E}$ is given by

$$
\omega_{\mathcal{E}}(e)\left(v \otimes e^{\prime}\right)=(-1)^{\operatorname{deg} e\left(\operatorname{deg} v+\operatorname{deg} e^{\prime}\right)} v \otimes e^{\prime \iota} e
$$

for $e, e^{\prime} \in \mathcal{E}$ and $v \in V$.

We let $\mathcal{A}$ act on $V \otimes_{\mathcal{D}} \mathcal{E}$ in the first variable, and $K$ in both variables. In other words,

$$
\pi(a)=\pi_{V}(a) \otimes \mathbf{1} ; \quad \nu(k)=\nu_{V}(k) \otimes \chi_{\mathcal{E}}(k)
$$

for $a \in \mathcal{A}$ and $k \in K$. It is straightforward to see that these actions are well defined, and that in this way $V \otimes_{\mathcal{D}} \mathcal{E}$ becomes an $(\mathcal{A}, K, \mathcal{E})$-module.

Clearly, in this way we have defined a functor $V \mapsto V \otimes_{\mathcal{D}} \mathcal{E}$ from $\mathcal{M}(\mathcal{A}, K, \mathcal{D})$ into $\mathcal{M}(\mathcal{A}, K, \mathcal{E})$. This functor is left adjoint to For. The adjunction morphisms are given as follows. For an $(\mathcal{A}, K, \mathcal{D})$-module $V, \Phi_{V}: V \rightarrow V \otimes_{\mathcal{D}} \mathcal{E}$ is given by

$$
\Phi_{V}(v)=v \otimes 1 .
$$

For an $(\mathcal{A}, K, \mathcal{E})$-module $W, \Psi_{W}: W \otimes_{\mathcal{D}} \mathcal{E} \rightarrow W$ is given by

$$
\Psi_{W}(w \otimes e)=(-1)^{\operatorname{deg} w \operatorname{deg} e} \omega_{W}\left({ }^{\iota} e\right) w .
$$

It is easy to see that these morphisms are well defined, that $\Phi_{V}$ is an $(\mathcal{A}, K, \mathcal{D})$ morphism and that $\Psi_{W}$ is an $(\mathcal{A}, K, \mathcal{E})$-morphism, and that in this way we really get adjunction. So we have

2.5.1. Theorem. The functor $V \mapsto V \otimes_{\mathcal{D}} \mathcal{E}$ described above is left adjoint to the forgetful functor from $\mathcal{M}(\mathcal{A}, K, \mathcal{E})$ into $\mathcal{M}(\mathcal{A}, K, \mathcal{D})$. The same is true on the level of homotopic categories. 
Proof. It remains to prove the claim about homotopic categories. It is clear that $-\otimes_{\mathcal{D}} \mathcal{E}$ is well defined on the level of graded modules. To check that this functor commutes with translation, we use the obvious identification of $T(V) \otimes_{\mathcal{D}} \mathcal{E}$ and $T\left(V \otimes_{\mathcal{D}} \mathcal{E}\right)$. The actions of $\mathcal{A}$ and $K$ obviously agree, and it is straightforward to check that the action of $\mathcal{E}$ and the differential agree as well.

To apply 2.4.1, it remains to show that our functor induces morphisms of complexes between corresponding Hom -complexes. However, this follows from 2.4.2(ii).

Remark. It would look more natural to consider the similarly defined functor $V \mapsto$ $\mathcal{E} \otimes_{\mathcal{D}} V$ instead of the above functor. This would eliminate passing from left to right modules and back. On the other hand, in the usual definition of the standard complex of a Lie algebra the $i_{\xi}$ 's come from the right multiplication. We do not want to change this, and one of the important examples will be $\mathcal{D}=\mathcal{U}(\mathfrak{k}), \mathcal{E}=N(\mathfrak{k})$. Hence we consider $V \otimes_{\mathcal{D}} \mathcal{E}$, with $\mathcal{E}$-action given by right multiplication.

As usual, one can construct the right adjoint of For in a similar way, using a Hom-space instead of the tensor product. We omit this construction since it is not needed in this paper.

2.5.2. Examples. Here are the two most important cases of the above constructions for our applications.

(i) $\mathcal{D}=\mathcal{U}(\mathfrak{k})$ and $\mathcal{E}=N(\mathfrak{k})$. Then $\mathcal{M}(\mathcal{A}, K, \mathcal{U}(\mathfrak{k}))$ is just the category $C\left(\mathcal{M}(\mathcal{A}, K)_{w}\right)$ of complexes of weak $(\mathcal{A}, K)$-modules, and $\mathcal{M}(\mathcal{A}, K, N(\mathfrak{k}))$ is the category $C(\mathcal{A}, K)$ of equivariant $(\mathcal{A}, K)$-complexes. For is just the obvious forgetful functor, and the left adjoint of For is

$$
V \longmapsto C_{\mathfrak{k}}(V)=V \otimes_{\mathcal{U}(\mathfrak{k})} N(\mathfrak{k}) .
$$

Since $N(\mathfrak{k})$ is a free $\mathcal{U}(\mathfrak{k})$-module for the left multiplication, this functor preserves acyclicity, i.e., if a complex of weak $(\mathcal{A}, K)$-modules $V$ is acyclic, then $C_{\mathfrak{k}}(V)$ is also acyclic.

(For has also a right adjoint $C^{\mathfrak{k}}=\operatorname{Hom}_{\mathcal{U}(\mathfrak{k})}(N(\mathfrak{k}),-$ ), which we will not need.)

(ii) $\mathcal{D}=N(\mathfrak{k}), \mathcal{E}=\mathbb{C}$ and $\alpha: N(\mathfrak{k}) \rightarrow \mathbb{C}$ is the counit map (see 2.2.4). Then $\mathcal{M}(\mathcal{A}, K, N(\mathfrak{k}))=C(\mathcal{A}, K)$ as in (i), while $\mathcal{M}(\mathcal{A}, K, \mathbb{C})$ is the category $C(\mathcal{M}(\mathcal{A}, K))$ of complexes of $(\mathcal{A}, K)$-modules. The corresponding forgetful functor can be described in the following way: A complex $V$ of $(\mathcal{A}, K)$ modules can be viewed as an equivariant $(\mathcal{A}, K)$-complex, if we define all $i_{\xi}$ 's to be 0 . We will denote this functor by $Q$. The left adjoint of $Q$ is the functor

$$
V \longmapsto V \otimes_{N(\mathfrak{k})} \mathbb{C},
$$

i.e., the functor of taking $\underline{\underline{k}}$-coinvariants. This functor plays an important role in $[\mathrm{P} 2$. (Similarly, the right adjoint of $Q$ is the functor of taking k-invariants.)

2.6. A property of $\mathbf{K}$-projectives. Let us recall that an $(\mathcal{A}, K, \mathcal{D})$-module $V$ is called K-projective, if for any acyclic $(\mathcal{A}, K, \mathcal{D})$-module $W, \operatorname{Hom}_{K(\mathcal{A}, K, \mathcal{D})}(V, W)=$ 0 . Dually, one defines K-injective $(\mathcal{A}, K, \mathcal{D})$-modules: $V$ is K-injective if for any acyclic $(\mathcal{A}, K, \mathcal{D})$-module $W, \operatorname{Hom}_{K(\mathcal{A}, K, \mathcal{D})}(W, V)=0$.

Since any translate of an acyclic object is acyclic, one sees that $V$ is K-projective if and only if for any acyclic $(\mathcal{A}, K, \mathcal{D})$-module $W$, the complex $\operatorname{Hom}_{K(\mathcal{A}, K, \mathcal{D})}(V, W)$ 
is acyclic. A similar claim holds for K-injectives. Also, the K-projectives form a null system in $K(\mathcal{A}, K, \mathcal{D})$ (see [KS], Definition 1.6.6). This follows immediately from the definitions. The same is true for K-injectives.

The purpose of this section is to prove a property of K-projectives, which is actually a property of any null system. This property is proved in 2.6 .4 below. It says that if an $(\mathcal{A}, K, \mathcal{D})$-module $V$ has a finite filtration such that the corresponding graded modules are K-projective, and if this filtration satisfies a certain splitting condition, then $V$ is K-projective. We will need this property in Section 4.2 to show that the standard complex $N(\mathfrak{k})$ is K-projective in a certain category. This will imply that equivariant Zuckerman functors, which we define first on the level of homotopic categories, actually make sense on the level of equivariant derived categories.

The property we need depends on certain properties of the cone, which seem to be interesting in themselves. First we define the notion of a semisplit short exact sequence (see $\underline{\mathrm{Sp}})$. A short exact sequence

$$
0 \rightarrow U \rightarrow V \rightarrow W \rightarrow 0
$$

in $\mathcal{M}(\mathcal{A}, K, \mathcal{D})$ is semisplit if it is split in the category of graded $(\mathcal{A}, K, \mathcal{D})$-modules. In other words, we can identify $V$ with $W \oplus U$ as a graded module, but the inclusion of $W$ is not necessarily a morphism of complexes. Clearly, for a morphism $f: X \rightarrow$ $Y$ in $\mathcal{M}(\mathcal{A}, K, \mathcal{D})$, the short exact sequence

$$
0 \rightarrow Y \stackrel{i_{f}}{\rightarrow} C_{f} \stackrel{p_{f}}{\rightarrow} T(X) \rightarrow 0
$$

is semisplit. We are going to show that any semisplit sequence arises in this way.

Let $0 \rightarrow U \rightarrow V \rightarrow W \rightarrow 0$ be a semisplit short exact sequence. Then the matrix of the differential of $V$ with respect to the decomposition $V=W \oplus U$ is

$$
d_{V}=\left(\begin{array}{cc}
d_{W} & 0 \\
\delta & d_{U}
\end{array}\right)
$$

where $\delta: W \rightarrow U$ is a linear map of degree 1 . We denote by the same letter the corresponding linear map from $W$ into $T(U)$ of degree 0 .

2.6.1. Lemma. The map $\delta: W \rightarrow T(U)$ is a morphism in $\mathcal{M}(\mathcal{A}, K, \mathcal{D})$.

Proof. It is clear that $\delta$ is an $(\mathcal{A}, K)$-morphism. To see it is a $\mathcal{D}$-morphism, we first note that for any $x \in \mathcal{D}$,

$$
\omega_{V}(x)=\left(\begin{array}{cc}
\omega_{W}(x) & 0 \\
0 & \omega_{U}(x)
\end{array}\right)
$$

since $V=W \oplus U$ as a $\mathcal{D}$-module. Let $v=\left(\begin{array}{c}w \\ u\end{array}\right) \in V$. Since $V$ is a DG module over $\mathcal{D}$, we have

$$
d_{V}\left(\omega_{V}(x) v\right)=\omega_{V}\left(d_{\mathcal{D}} x\right) v+(-1)^{\operatorname{deg} x} \omega_{V}(x) d_{V}(v),
$$

which becomes

$$
\left(\begin{array}{c}
d_{W}\left(\omega_{W}(x) w\right) \\
\delta\left(\omega_{W}(x) w\right)+d_{U}\left(\omega_{U}(x) u\right)
\end{array}\right)=\left(\begin{array}{c}
\omega_{W}\left(d_{\mathcal{D}} x\right) w+(-1)^{\operatorname{deg} x} \omega_{W}(x) d_{W}(w) \\
\omega_{U}\left(d_{\mathcal{D}} x\right) u+(-1)^{\operatorname{deg} x}\left(\omega_{U}(x) \delta w+\omega_{U}(x) d_{U} u\right)
\end{array}\right)
$$

in the matrix representation. Since $W$ and $U$ are also DG modules over $\mathcal{D}$, this implies

so $\delta$ is indeed a $\mathcal{D}$-morphism.

$$
\delta \omega_{W}(x)=(-1)^{\operatorname{deg} x} \omega_{U}(x) \delta=\omega_{T(U)}(x) \delta,
$$


It remains to show that $\delta$ is a morphism of complexes. However, $d_{V}^{2}=0$, i.e.,

$$
\left(\begin{array}{cc}
d_{W}^{2} & 0 \\
\delta d_{W}+d_{U} \delta & d_{U}^{2}
\end{array}\right)=0
$$

hence

$$
\delta d_{W}=-d_{U} \delta=d_{T(U)} \delta
$$

This finishes the proof.

Now we see that $V$ is the cone of the morphism $T^{-1}(\delta): T^{-1}(W) \rightarrow U$. Namely, $V=T\left(T^{-1}(W)\right) \oplus U$ as a graded module, and its differential is $\left(\begin{array}{cc}d_{T\left(T^{-1} W\right)} & 0 \\ T\left(T^{-1} \delta\right) & d_{U}\end{array}\right)$, which is equal to $d_{T^{-1} \delta}$. Therefore we have proved the following characterization of the cone.

2.6.2. Proposition. Let $0 \rightarrow U \rightarrow V \rightarrow W \rightarrow 0$ be a semisplit short exact sequence in $\mathcal{M}(\mathcal{A}, K, \mathcal{D})$. Then any choice of a splitting for this sequence exhibits $V$ as the cone of a morphism from $T^{-1}(W)$ into $U$.

This has the following immediate consequences, which are useful for proving that certain objects are K-projective or K-injective (see 3.2.6).

2.6.3. Corollary. Let $0 \rightarrow U \rightarrow V \rightarrow W \rightarrow 0$ be a semisplit short exact sequence in $\mathcal{M}(\mathcal{A}, K, \mathcal{D})$. Let $\mathcal{N}$ be a null system in $K(\mathcal{A}, K, \mathcal{D})$. Then if any two of the objects $U, V$ and $W$ are in $\mathcal{N}$, so is the third.

Proof. From 2.6.2 we see that we have a distinguished triangle

$$
T^{-1}(W) \rightarrow U \rightarrow V \rightarrow W
$$

in $K(\mathcal{A}, K, \mathcal{D})$. Therefore the claim follows from the properties (N2) and (N3) in the definition of a null system ([KS], Definition 1.6.6).

2.6.4. Corollary. Let $\mathcal{N}$ be a null system in $K(\mathcal{A}, K, \mathcal{D})$ and let $V$ be an $(\mathcal{A}, K, \mathcal{D})$ module. Assume

$$
0=F_{0} V \subset F_{1} V \subset \cdots \subset F_{n} V=V
$$

is a finite filtration of $V$ by $(\mathcal{A}, K, \mathcal{D})$-submodules, such that the graded objects

$$
G r_{i} V=F_{i} V / F_{i-1} V, \quad i=1, \ldots, n,
$$

belong to $\mathcal{N}$. Assume further that $F_{i} V$ is isomorphic to $F_{i-1} V \oplus G r_{i} V$ as a graded $(\mathcal{A}, K, \mathcal{D})$-module. Then $V$ belongs to $\mathcal{N}$.

Proof. Starting from $0 \in \mathcal{N}$ and using 2.6.3, we prove inductively that $F_{i} V$ belong to $\mathcal{N}$ for all $i$. In particular, for $i=n$ we get that $V$ belongs to $\mathcal{N}$.

It is possible to generalize 2.6.4 to infinite filtrations, both increasing and decreasing (for decreasing filtrations, one needs a finiteness assumption). These generalizations together with the results of 2.5 lead to constructions of enough K-injectives in $K(\mathcal{A}, K, \mathcal{D})$, and also $\mathrm{K}$-projectives in case $K$ is reductive. The construction of $\mathrm{K}$ injectives was done in BL2, 1.15.3, in essentially the same way. Both constructions are explained in detail in $[\mathrm{P} 1], \S 5.6$. 


\section{Equivariant ZuCKERMAN FunCtORS}

In this section we consider a situation as in Section 1.3: $(\mathcal{A}, K)$ is a HarishChandra pair, and $\gamma: T \rightarrow K$ is a morphism of algebraic groups. We will assume that the differential of $\gamma$ is injective, so that the Lie algebra $\mathfrak{t}$ of $T$ can be viewed as a Lie subalgebra of the Lie algebra $\mathfrak{k}$ of $K$, and $N(\mathfrak{t})$ can be viewed as a DG subalgebra of $N(\mathfrak{k})$. Note that $(\mathcal{U}(\mathfrak{k}), T)$ is another Harish-Chandra pair in the obvious way.

We are going to construct the right adjoint $\Gamma_{K, T}^{e q}$ of the forgetful functor from the category of equivariant $(\mathcal{A}, K)$-complexes (i.e., $(\mathcal{A}, K, N(\mathfrak{k}))$-modules) into the category of equivariant $(\mathcal{A}, T)$-complexes (i.e., $(\mathcal{A}, T, N(\mathfrak{t}))$-modules). $\Gamma_{K, T}^{e q}$ will also define a functor between the corresponding homotopic categories. Furthermore, we will show that, in case $T$ is reductive, this functor is "acyclic" (i.e., preserves acyclic complexes, or equivalently, preserves quasi-isomorphisms). It follows that this functor defines a functor between the corresponding equivariant derived categories, denoted again by $\Gamma_{K, T}^{e q}$. This is the equivariant analogue of the derived Zuckerman functor. Finally, $\Gamma_{K, T}^{e q}$ is given on all levels by the same explicit formula (not involving resolutions), analogous to the Duflo-Vergne formula from 1.3.3.

We will further show that in case $\mathcal{A}$ is a flat $\mathcal{U}(\mathfrak{k})$-module for the right multiplication, the cohomology modules of $\Gamma_{K, T}^{e q}(V)$, for an $(\mathcal{A}, T)$-module $V$ viewed as an equivariant complex concentrated in degree 0 , are equal to the classical derived Zuckerman functors of $V$.

One advantage of the equivariant construction over the classical one is the fact that the equivariant construction is independent of $\mathcal{A}$; in particular, if $(\mathfrak{g}, K)$ is a Harish-Chandra pair, then the same formula describes the adjoint for $\mathcal{A}=\mathcal{U}(\mathfrak{g})$ and for $\mathcal{A}=\mathcal{U}_{\theta}$, the quotient of $\mathcal{U}(\mathfrak{g})$ corresponding to an infinitesimal character. This leads to a natural way of localizing the Zuckerman construction; see [MP1], $\S 4$, and [MP2].

3.1. Construction of equivariant Zuckerman functors. Let $V$ be an equivariant $(\mathcal{A}, T)$-complex, i.e., an $(\mathcal{A}, T, N(\mathfrak{t}))$-module. Let $\pi_{V}, \nu_{V}$ and $\omega_{V}$ be the corresponding actions. We consider $V$ as a complex of weak $(\mathcal{A}, T)$-modules and apply to it the functor $\operatorname{Ind}_{w}$ from Section 1.3. In this way we get a complex of weak $(\mathcal{A}, K)$-modules $\operatorname{Ind}_{w}(V)=R(K, V)=R(K) \otimes_{\mathbb{C}} V$. We denote the corresponding actions of $\mathcal{A}$ and $K$ by $\pi_{R}$ and $\nu_{R}$; recall that $\nu_{R}$ is just the right translation, and that for $a \in \mathcal{A}$,

$$
\left(\pi_{R}(a) F\right)(k)=\pi_{V}\left(\phi_{K}(k) a\right)(F(k)), \quad k \in K .
$$

Both of these are actions by morphisms of complexes. Note that the differential of $R(K, V)$ is induced by the differential of $V$.

Now we want to construct a $(\mathfrak{k}, T, N(\mathfrak{t}))$-action on $R(K, V)$. We already constructed actions $\lambda_{\mathfrak{k}}$ of $\mathfrak{k}$ and $\lambda_{T}$ of $T$ in Section 1.3; they were both given as the tensor product of the left regular action on $R(K)$ with the given action on $V$. Clearly, in our present situation when $V$ is a complex, they are actions by morphisms of complexes (namely, the actions $\pi_{V}$ and $\nu_{V}$ are by morphisms of complexes). Therefore, $R(K, V)$ is a complex of weak $(\mathfrak{k}, T)$-modules with respect to $\lambda_{\mathfrak{k}}$ and $\lambda_{T}$.

We define an action of $N(\mathfrak{t})$ on $R(K, V)$ by

$$
\left(\lambda_{N}(n) F\right)(k)=\omega_{V}(n)(F(k)), \quad k \in K
$$


for $n \in N(\mathfrak{t})$ and $F \in R(K, V)$. In other words, $\lambda_{N}$ is the tensor product of the trivial action of $N(\mathfrak{t})$ on $R(K)$ with the given action on $V$. It is clearly a DG action.

The following lemma is straightforward.

3.1.1. Lemma. Let $V$ be an $(\mathcal{A}, T, N(\mathfrak{t}))$-module. Then

(i) With the above described action $\lambda, \operatorname{Ind}_{w}(V)=R(K, V)$ is a $(\mathfrak{k}, T, N(\mathfrak{t}))$ module.

(ii) The actions $\pi_{R}$ and $\nu_{R}$ of $\mathcal{A}$ and $K$ on $R(K, V)$ commute with the $\lambda$-action.

(iii) Let $\varphi: V \rightarrow W$ be a morphism of $(\mathcal{A}, T, N(\mathfrak{t}))$-modules. Since $\varphi$ is a morphism of complexes of weak $(\mathcal{A}, T)$-modules, we can consider the morphism $\operatorname{Ind}_{w}(\varphi)$ of complexes of weak $(\mathcal{A}, K)$-modules. Then $\operatorname{Ind}_{w}(\varphi)$ intertwines the $\lambda$-actions.

Consider now the standard complex $N(\mathfrak{k})$ of $\mathfrak{k}$ as a $(\mathfrak{k}, T, N(\mathfrak{t}))$-module, in the following way. $\mathfrak{k}$ acts by $\pi_{N}$ which is the left multiplication, $T$ acts by the action $\nu_{N}$ induced by $\phi$, and $N(\mathfrak{t})$ acts by $\omega_{N}$ which is the right multiplication twisted to a left action:

$$
\omega_{N}(m) n=(-1)^{\operatorname{deg} m \operatorname{deg} n} n^{\iota} m
$$

for $m \in N(\mathfrak{t})$ and $n \in N(\mathfrak{k})$. Here $\iota$ is the principal anti-automorphism of $N(\mathfrak{t})$, which is the same as the restriction of the principal anti-automorphism of $N(\mathfrak{k})$. See Section 2.2, in particular 2.2.3. It is straightforward to check that in this way $N(\mathfrak{k})$ indeed becomes a $(\mathfrak{k}, T, N(\mathfrak{t}))$-module. We define

$$
\Gamma_{K, T}^{e q}(V)=\operatorname{Hom}_{(\mathfrak{k}, T, N(\mathfrak{t}))}(N(\mathfrak{k}), R(K, V))
$$

where the $(\mathfrak{k}, T, N(\mathfrak{t}))$-action on $R(K, V)$ is the $\lambda$-action. $\Gamma_{K, T}^{e q}(V)$ is a complex of vector spaces as we saw at the beginning of Section 2.4.

Recall from Section 2.4 that the condition for $f: N(\mathfrak{k}) \rightarrow R(K, V)$ to be an $N(\mathfrak{t})$-morphism is

$$
f\left(\omega_{N}(m) n\right)=(-1)^{\operatorname{deg} f \operatorname{deg} m} \lambda_{N}(m)(f(n)) .
$$

We define actions of $\mathcal{A}, K$ and $N(\mathfrak{k})$ on $f \in \Gamma_{K, T}^{e q}(V)$ as follows:

$$
\begin{gathered}
\pi_{\Gamma}(a) f=\pi_{R}(a) \circ f, \quad a \in \mathcal{A}, \\
\nu_{\Gamma}(k) f=\nu_{R}(k) \circ f, \quad k \in K, \\
\left(\omega_{\Gamma}(n) f\right)(m)(k)=(-1)^{\operatorname{deg} n \operatorname{deg} f} f\left(\operatorname{Ad} k\left({ }^{\iota} n\right) m\right)(k)
\end{gathered}
$$

for $a \in \mathcal{A}, m, n \in N(\mathfrak{k})$ and $k \in K$.

3.1.2. Theorem. With the above actions, $\Gamma_{K, T}^{e q}(V)$ is an $(\mathcal{A}, K, N(\mathfrak{k}))$-module. Furthermore, $\Gamma_{K, T}^{e q}$ is a functor from $\mathcal{M}(\mathcal{A}, T, N(\mathfrak{t}))$ into $\mathcal{M}(\mathcal{A}, K, N(\mathfrak{k}))$.

Proof. It is clear from 3.1.1 that $\pi_{\Gamma}$ and $\nu_{\Gamma}$ are well-defined actions by morphisms of complexes. It is also clear that $\nu_{\Gamma}$ is algebraic, and that $\pi_{\Gamma}$ is $\nu_{\Gamma}$-equivariant.

Let us prove that the action $\omega_{\Gamma}$ is a well-defined DG action. Let $f \in \Gamma_{K, T}^{e q}(V)$ and $n \in N(\mathfrak{k})$. First, it is obvious that $\omega_{\Gamma}(n) f$ is a linear map of degree $\operatorname{deg} n+\operatorname{deg} f$. We have to prove that $\omega_{\Gamma}(n) f$ is a $(\mathfrak{k}, T, N(\mathfrak{t}))$-morphism; then it will be clear that $\omega_{\Gamma}$ is a DG action. Namely, it is clear that $(f n)(m)=f(n m), n, m \in N(\mathfrak{k})$, $f \in \Gamma_{K, T}^{e q}(V)$, defines a right DG action of $N(\mathfrak{k})$. Hence the same is true for

$$
(f n)(m)(k)=f(\operatorname{Ad} k(n) m)(k), \quad k \in K,
$$

and so, by $2.2 .3, \omega_{\Gamma}$ is a left DG action of $N(\mathfrak{k})$. 
It is straightforward to check that $\omega_{\Gamma}(n) f$ is a $T$-morphism and an $N(\mathfrak{t})$ morphism. To see that it is also a $\mathfrak{k}$-morphism, let $X \in \mathfrak{k}$. Then

$$
\begin{aligned}
& \lambda(X)\left(\left(\omega_{\Gamma}(n) f\right)(m)\right)(k)=\pi_{V}(X)\left(\left(\omega_{\Gamma}(n) f\right)(m)(k)\right)+L_{X}\left(\left(\omega_{\Gamma}(n) f\right)(m)\right)(k) \\
& =(-1)^{\operatorname{deg} n \operatorname{deg} f} \pi_{V}(X)\left(f\left(\operatorname{Ad} k\left({ }^{l} n\right) m\right)(k)\right)+\left.\frac{d}{d t}\left(\left(\omega_{\Gamma}(n) f\right)(m)\right)(\exp (-t X) k)\right|_{t=0} \\
& =(-1)^{\operatorname{deg} n \operatorname{deg} f}\left(\pi_{V}(X)\left(f\left(\operatorname{Ad} k\left({ }^{\iota} n\right) m\right)(k)\right)\right. \\
& \left.+\left.\frac{d}{d t} f\left(\operatorname{Ad}(\exp (-t X) k)\left({ }^{\iota} n\right) m\right)(\exp (-t X) k)\right|_{t=0}\right) \\
& =(-1)^{\operatorname{deg} n \operatorname{deg} f}\left(\pi_{V}(X)\left(f\left(\operatorname{Ad} k\left({ }^{l} n\right) m\right)(k)\right)\right. \\
& \left.+f\left(-\left[X, \operatorname{Ad} k\left({ }^{\iota} n\right)\right] m\right)(k)+\left.\frac{d}{d t} f\left(\operatorname{Ad} k\left({ }^{\iota} n\right) m\right)(\exp (-t X) k)\right|_{t=0}\right) \\
& =(-1)^{\operatorname{deg} n \operatorname{deg} f}\left(f\left(X \operatorname{Ad} k\left({ }^{\iota} n\right) m\right)(k)-f\left(\left[X, \operatorname{Ad} k\left({ }^{\iota} n\right)\right] m\right)(k)\right) \\
& =(-1)^{\operatorname{deg} n \operatorname{deg} f} f\left(\operatorname{Ad} k\left({ }^{l} n\right) X m\right)(k)=\left(\omega_{\Gamma}(n) f\right)(X m)(k) \text {, }
\end{aligned}
$$

for any $m \in N(\mathfrak{k})$ and $k \in K$. Here we used the fact that since $f$ is a $\mathfrak{k}$-morphism,

$$
\begin{aligned}
& \pi_{V}(X)\left(f\left(\operatorname{Ad} k\left({ }^{\iota} n\right) m\right)(k)\right)+\left.\frac{d}{d t} f\left(\operatorname{Ad} k\left({ }^{\iota} n\right) m\right)(\exp (-t X) k)\right|_{t=0} \\
& =f\left(X \operatorname{Ad} k\left({ }^{\iota} n\right) m\right)(k),
\end{aligned}
$$

for any (fixed) $k \in K$.

The $\nu_{\Gamma}$-equivariance of $\omega_{\Gamma}$ and the commuting of $\omega_{\Gamma}$ and $\pi_{\Gamma}$ are straightforward.

It remains to show that $\nu_{\Gamma}-\pi_{\Gamma}=\omega_{\Gamma}$ on $\mathfrak{k}$. This follows from 1.3.1, which says that for any $F \in R(K, V)$ and $X \in \mathfrak{k}$ we have

$$
\left(\nu_{R}(X) F\right)(k)-\left(\pi_{R}(X) F\right)(k)=\left(\omega_{R}(X) F\right)(k)=-\left(\lambda_{\mathfrak{k}}(\operatorname{Ad} k(X)) F\right)(k),
$$

for any $k \in K$.

This shows that $\Gamma_{K, T}^{e q}(V)$ is indeed an $(\mathcal{A}, K, N(\mathfrak{k}))$-module. It is now easy to check that $\Gamma_{K, T}^{e q}$ is a functor; its action on a morphism $\varphi: V \rightarrow W$ of $(\mathcal{A}, T, N(\mathfrak{t}))$ modules is given by

$$
\left(\Gamma_{K, T}^{e q}(\varphi)(f)\right)(n)(k)=\varphi(f(n)(k)),
$$

for $f \in \Gamma_{K, T}^{e q}(V), n \in N(\mathfrak{k})$ and $k \in K$.

3.1.3. Theorem. The functor $\Gamma_{K, T}^{e q}$ is right adjoint to the forgetful functor from the category $\mathcal{M}(\mathcal{A}, K, N(\mathfrak{k}))$ into the category $\mathcal{M}(\mathcal{A}, T, N(\mathfrak{t}))$.

Proof. We will just define the adjunction morphisms and leave the straightforward checking to the reader. For an $(\mathcal{A}, K, N(\mathfrak{k}))$-module $V$, we define $\Phi_{V}: V \rightarrow$ $\Gamma_{K, T}^{e q}(V)$ by

$$
\left(\Phi_{V}(v)(n)\right)(k)=(-1)^{\operatorname{deg} v \operatorname{deg} n} \omega_{V}\left({ }^{\iota} n\right) \nu_{V}(k) v,
$$

for $v \in V, n \in N(\mathfrak{k})$ and $k \in K$. For an $(\mathcal{A}, T, N(\mathfrak{t}))$-module $W$ we define $\Psi_{W}$ : $\Gamma_{K, T}^{e q}(W) \rightarrow W$ by

for $f \in \Gamma_{K, T}^{e q}(W)$.

$$
\Psi_{W}(f)=f(1)(1)
$$

Now we want to show that $\Gamma_{K, T}^{e q}$ defines an exact functor on the level of homotopic categories. The proof of this is essentially the same as the proof of 2.4.3. Namely, the proof of functoriality of $\Gamma_{K, T}^{e q}$ in 3.1 .2 shows also that $\Gamma_{K, T}^{e q}$ transforms 
graded $(\mathcal{A}, T, N(\mathfrak{t}))$-morphisms into graded $(\mathcal{A}, K, N(\mathfrak{k}))$-morphisms. On the other hand, it was proved in the proof of 2.4.3 that $\Gamma_{K, T}^{e q}$ satisfies the other conditions of 2.4.1; namely, these other conditions are independent of the $(\mathcal{A}, K, N(\mathfrak{k}))$-action and depend only on the structure of a complex of vector spaces.

So $\Gamma_{K, T}^{e q}$ indeed defines an exact functor between the homotopic categories, which we denote again by $\Gamma_{K, T}^{e q}$. By 2.4.4 (or 2.4.5), it is still adjoint to the forgetful functor on the homotopic level. Hence we have proved

3.1.4. Proposition. $\Gamma_{K, T}^{e q}$ defines an exact functor from the category $K(\mathcal{A}, T, N(\mathfrak{t}))$ into the category $K(\mathcal{A}, K, N(\mathfrak{k}))$. This functor is right adjoint to the forgetful functor.

To pass to derived categories, we assume that $T$ is reductive (for the case of non-reductive $T$, see MP1, $\S 2$ ). In that case, we are going to prove that $\Gamma_{K, T}^{e q}$ is an acyclic functor, i.e., transforms acyclic equivariant $(\mathcal{A}, T)$-complexes into acyclic equivariant $(\mathcal{A}, K)$-complexes. This implies that $\Gamma_{K, T}^{e q}$ also transforms quasiisomorphisms into quasi-isomorphisms, and hence defines a functor on the level of derived categories; this functor is equal to $\Gamma_{K, T}^{e q}$ on objects, and acts on triples in the obvious way.

To show that $\Gamma_{K, T}^{e q}$ is acyclic, note first that if $V$ is an acyclic equivariant $(\mathcal{A}, T)$ complex, then $R(K, V)$ is also acyclic, either as a complex of weak $(\mathcal{A}, K)$-modules, or as a $(\mathfrak{k}, T, N(\mathfrak{t}))$-module. On the other hand, acyclicity of $\Gamma_{K, T}^{e q}(V)$ can be checked on the level of vector spaces. Therefore, it is enough to show that the functor $\operatorname{Hom}_{(\mathfrak{k}, T, N(\mathfrak{t}))}(N(\mathfrak{k}),-)$ transforms acyclic $(\mathfrak{k}, T, N(\mathfrak{t}))$-modules into acyclic complexes of vector spaces.

In other words, we need to show that $N(\mathfrak{k})$ is a K-projective $(\mathfrak{k}, T, N(\mathfrak{t}))$-module (see §2.6). This will be proved in 3.2.6 below. Furthermore, $\Gamma_{K, T}^{e q}(V): D(\mathcal{A}, T, N(\mathfrak{t}))$ $\rightarrow D(\mathcal{A}, K, N(\mathfrak{k}))$ remains adjoint to the forgetful functor. This is a quite general fact about deriving adjoint functors (see [M], Chapter 5, Theorem 1.6.1); our situation with both functors acyclic is comparatively simple. So once we prove 3.2.6, we will get the following theorem.

3.1.5. Theorem. Assume $T$ is reductive. Then the functor $\Gamma_{K, T}^{e q}$ is acyclic and hence defines the same named functor on the level of derived categories, from $D(\mathcal{A}, T, N(\mathfrak{t}))$ into $D(\mathcal{A}, K, N(\mathfrak{k}))$. This functor is right adjoint to the forgetful functor.

3.2. K-projectivity of $N(\mathfrak{k})$. To prove that $N(\mathfrak{k})$ is a K-projective $(\mathfrak{k}, T, N(\mathfrak{t}))$ module, we use the Hochschild-Serre filtration (see [HS]), which we now describe. To construct this filtration we do not need $T$ to be reductive; this will be required only in the proof of K-projectivity.

Let $p$ be an integer between 0 and $\operatorname{dim} \mathfrak{k}$. We consider $\bigoplus_{i=0}^{p} N^{-i}(\mathfrak{k})$. This is clearly a subcomplex and a $(\mathfrak{k}, T)$-submodule of $N(\mathfrak{k})$; however, it is not an $N(\mathfrak{t})$ submodule. Therefore we define

$$
F_{p} N(\mathfrak{k})=\omega_{N}(N(\mathfrak{t}))\left(\bigoplus_{i=0}^{p} N^{-i}(\mathfrak{k})\right) .
$$

This is now clearly an $N(\mathfrak{t})$-submodule; however, it is also a $\mathfrak{k}$-submodule since $\pi_{N}$ and $\omega_{N}$ commute, and a $T$-submodule since $\omega_{N}$ is $T$-equivariant and $N(\mathfrak{t})$ is $T$-invariant. Finally, it is a subcomplex by the DG property of $\omega_{N}$. Hence we have defined an increasing filtration of $N(\mathfrak{k})$ by $(\mathfrak{k}, T, N(\mathfrak{t}))$-submodules. 
In the following lemma we describe $F_{p} N(\mathfrak{k})$ more explicitly. In particular, from this description it will become clear that our filtration is really the Hochschild-Serre filtration. The proof is straightforward.

3.2.1. Lemma. For $k \leq p, F_{p} N^{-k}(\mathfrak{k})=N^{-k}(\mathfrak{k})$. For $k \geq p$,

$$
F_{p} N^{-k}(\mathfrak{k})=N^{-p}(\mathfrak{k}) \bigwedge^{k-p}(\mathfrak{t}) .
$$

Here the right-hand side is a subspace of $N^{-k}(\mathfrak{k})$ via multiplication in $N(\mathfrak{k})$.

In this description of the filtration, the containment $F_{p} N(\mathfrak{k}) \subset F_{p+1} N(\mathfrak{k})$ becomes

$$
N^{-p}(\mathfrak{k}) \bigwedge^{k-p}(\mathfrak{t}) \subset N^{-p-1}(\mathfrak{k}) \bigwedge^{k-p-1}(\mathfrak{t}),
$$

the inclusion being given in the obvious way, i.e., by

$$
(u \otimes \xi)\left(\eta_{1} \wedge \cdots \wedge \eta_{k-p}\right)=\left(u \otimes \xi \wedge \eta_{1}\right)\left(\eta_{2} \wedge \cdots \wedge \eta_{k-p}\right) .
$$

Furthermore, it is clear that $F_{\operatorname{dim} \mathfrak{k}} N(\mathfrak{k})=N(\mathfrak{k})$, while $F_{0} N(\mathfrak{k})=\mathcal{U}(\mathfrak{k}) \bigwedge(\mathfrak{t})$ can be identified with $\mathcal{U}(\mathfrak{k}) \otimes_{\mathbb{C}} \wedge(\mathfrak{t})$. We define $F_{-1} N(\mathfrak{k})=0$.

We want to identify the graded object corresponding to this filtration. For this we need the following lemma about exterior algebras, the proof of which follows easily from the results in [Bo, III.7.

3.2.2. Lemma. Let $\mathfrak{k}$ be a finite dimensional vector space and $\mathfrak{t}$ a subspace of $\mathfrak{k}$. Denote by $\pi$ the canonical graded map from $\bigwedge(\mathfrak{k})$ into $\bigwedge(\mathfrak{k} / \mathfrak{t})$ induced by the projection $\mathfrak{k} \rightarrow \mathfrak{k} / \mathfrak{t}$. Then

(i) $\pi$ is surjective and its kernel is $\bigwedge(\mathfrak{k}) \mathfrak{t}$, the ideal in $\bigwedge(\mathfrak{k})$ generated by $\mathfrak{t}$.

(ii) The bigraded linear map

$$
m: \bigwedge(\mathfrak{k}) \otimes_{\mathbb{C}} \bigwedge(\mathfrak{t}) \rightarrow \bigwedge(\mathfrak{k}) \bigwedge(\mathfrak{t})
$$

given by multiplication is surjective and its kernel is contained in $\bigwedge(\mathfrak{k}) \mathfrak{t} \otimes_{\mathbb{C}}$ $\bigwedge(\mathfrak{t})$.

(iii) The map $\pi^{i} \otimes 1: \bigwedge^{i}(\mathfrak{k}) \otimes_{\mathbb{C}} \bigwedge^{j}(\mathfrak{t}) \rightarrow \bigwedge^{i}(\mathfrak{k} / \mathfrak{t}) \otimes_{\mathbb{C}} \bigwedge^{j}(\mathfrak{t})$ defines a map

$$
\varphi: \bigwedge^{i}(\mathfrak{k}) \bigwedge^{j}(\mathfrak{t}) \rightarrow \bigwedge^{i}(\mathfrak{k} / \mathfrak{t}) \otimes_{\mathbb{C}} \bigwedge^{j}(\mathfrak{t})
$$

such that $\pi^{i} \otimes 1=\varphi \circ \mathrm{m}$. The kernel of $\varphi$ is

$$
\bigwedge^{i-1}(\mathfrak{k}) \bigwedge^{j+1}(\mathfrak{t})=\left(\bigwedge^{i-1}(\mathfrak{k}) \mathfrak{t}\right) \bigwedge^{j}(\mathfrak{t}) \subset \bigwedge^{i}(\mathfrak{k}) \bigwedge^{j}(\mathfrak{t}) .
$$

3.2.3. Remark. We are, of course, going to apply 3.2.2 in the case studied above, of a Lie algebra $\mathfrak{k}$ and its subalgebra $\mathfrak{t}$. In that case, $T$ acts on $\mathfrak{k}, \mathfrak{t}$ and $\mathfrak{k} / \mathfrak{t}$, and hence on the corresponding exterior algebras. It is then obvious that all the maps considered in 3.2.2 are $T$-morphisms. It is also clear that the maps $m$ and $\varphi$ from 3.2 .2 are morphisms with respect to the action of $\bigwedge(\mathfrak{t})$ via right multiplication.

We are ready now to describe the graded object corresponding to our filtration. Consider the algebraic $T$-module $\Lambda^{p}(\mathfrak{k} / \mathfrak{t})$ as a weak $(\mathbb{C}, T)$-module. We can apply the functor $\operatorname{ind}_{\mathcal{U}(\mathfrak{k}), \mathbb{C}}$ from Section 1.2 to this module. In this way we get a weak $(\mathfrak{k}, T)$-module

$$
\operatorname{ind}_{\mathcal{U}(\mathfrak{k}), \mathbb{C}} \bigwedge^{p}(\mathfrak{k} / \mathfrak{t})=\mathcal{U}(\mathfrak{k}) \otimes_{\mathbb{C}} \bigwedge^{p}(\mathfrak{k} / \mathfrak{t})
$$

recall that $\mathfrak{k}$ acts on this module via left multiplication in the first factor, while $T$ acts on both factors. Now we can consider $\mathcal{U}(\mathfrak{k}) \otimes_{\mathbb{C}} \bigwedge^{p}(\mathfrak{k} / \mathfrak{t})[p]$; it is a complex of 
weak $(\mathfrak{k}, T)$-modules concentrated in degree $-p$. We now apply the functor $C_{\mathfrak{t}}$ from 2.5.2(i) to this complex. In this way we obtain a $(\mathfrak{k}, T, N(\mathfrak{t}))$-module,

$$
C_{\mathfrak{t}}\left(\operatorname{ind}_{\mathcal{U}(\mathfrak{k}), \mathbb{C}} \bigwedge^{p}(\mathfrak{k} / \mathfrak{t})[p]\right)=\left(\mathcal{U}(\mathfrak{k}) \otimes_{\mathbb{C}} \bigwedge^{p}(\mathfrak{k} / \mathfrak{t})[p]\right) \otimes_{\mathcal{U}(\mathfrak{t})} N(\mathfrak{t}) .
$$

Recall from $\S 2.5$ that here the tensor product is taken with respect to the right action of $\mathcal{U}(\mathfrak{t})$ on the first factor, which is the $\omega$-action twisted by $\iota$, and the left multiplication in the second factor. The action of $\mathfrak{k}$ on the above module is given by the left multiplication in the first factor, $T$ acts on all three factors, while $N(\mathfrak{t})$ acts on the third factor by right multiplication twisted by $\iota$. The differential is the tensor product differential, but since $\mathcal{U}(\mathfrak{k}) \otimes_{\mathbb{C}} \bigwedge^{p}(\mathfrak{k} / \mathfrak{t})[p]$ has zero differential, we see that

$$
d=(-1)^{p} 1 \otimes d_{N},
$$

where $d_{N}$ denotes the differential of $N(\mathfrak{t})$.

Finally, let us note that that the above complex is concentrated in degrees between $-p$ and $-p-\operatorname{dim} \mathbf{t}$.

3.2.4. Proposition. The graded object attached to the Hochschild-Serre filtration $F N(\mathfrak{k})$ is given by the above described modules:

$$
G r_{p} N(\mathfrak{k})=C_{\mathfrak{t}}\left(\operatorname{ind}_{\mathcal{U}(\mathfrak{k}), \mathbb{C}} \bigwedge^{p}(\mathfrak{k} / \mathfrak{t})[p]\right) .
$$

Proof. Let us first note that for $p \leq k \leq p+\operatorname{dim} \mathfrak{t}, C_{\mathfrak{t}}\left(\operatorname{ind}_{\mathcal{U}(\mathfrak{k}), \mathbb{C}} \wedge^{p}(\mathfrak{k} / \mathfrak{t})[p]\right)^{-k}$ can be identified with

$$
\mathcal{U}(\mathfrak{k}) \otimes_{\mathbb{C}} \bigwedge^{p}(\mathfrak{k} / \mathfrak{t}) \otimes_{\mathbb{C}} \bigwedge^{k-p}(\mathfrak{t})
$$

In this interpretation, the actions of $\mathfrak{k}, T$ and $\underline{\mathfrak{t}}^{-1}$ are given in the obvious way (as before), while the action of $X \in \underline{t}^{0}$ is still by right multiplication, but we have to use the commuting rules as follows:

$$
\omega(X)(u \otimes \lambda \otimes \mu)=-u X \otimes \lambda \otimes \mu+u \otimes[X, \lambda] \otimes \mu+u \otimes \lambda \otimes[X, \mu] .
$$

Let us define a graded linear map $f: F_{p} N(\mathfrak{k}) \rightarrow C_{\mathfrak{t}}\left(\operatorname{ind}_{\mathcal{U}(\mathfrak{k}), \mathbb{C}} \bigwedge^{p}(\mathfrak{k} / \mathfrak{t})[p]\right)$ as follows: For $k<p, f^{-k}=0$, while for $k \geq p$,

$$
f^{-k}: \mathcal{U}(\mathfrak{k}) \otimes_{\mathbb{C}} \bigwedge^{p}(\mathfrak{k}) \bigwedge^{k-p}(\mathfrak{t}) \rightarrow \mathcal{U}(\mathfrak{k}) \otimes_{\mathbb{C}} \bigwedge^{p}(\mathfrak{k} / \mathfrak{t}) \otimes_{\mathbb{C}} \bigwedge^{k-p}(\mathfrak{t})
$$

is $1 \otimes \varphi$, where $\varphi: \bigwedge^{p}(\mathfrak{k}) \bigwedge^{k-p}(\mathfrak{t}) \rightarrow \bigwedge^{p}(\mathfrak{k} / \mathfrak{t}) \otimes_{\mathbb{C}} \bigwedge^{k-p}(\mathfrak{t})$ is the map from 3.2.2(iii). Here we used the description of $F_{p} N(\mathfrak{k})$ given by 3.2.1.

By 3.2.2(iii), $f$ is surjective and its kernel is equal to

$$
\mathcal{U}(\mathfrak{k}) \otimes_{\mathbb{C}} \bigwedge^{p-1}(\mathfrak{k}) \bigwedge^{k-p+1}(\mathfrak{t})
$$

in degrees $-k$, for $k \geq p$. It is clear that for $k<p$, ker $f^{-k}=N^{-k}(\mathfrak{k})$. So, by 3.2 .1 , the kernel of $f$ is exactly $F_{p-1} N(\mathfrak{k})$. Therefore to prove the theorem, it only remains to show that $f$ is a morphism of $(\mathfrak{k}, T, N(\mathfrak{t}))$-modules.

It is obvious that $f$ intertwines the $\mathfrak{k}$-actions. By 3.2.3 it also intertwines the $T$-actions and the actions of $\underline{\mathfrak{t}}^{-1}$. To see that it also intertwines the actions of $\underline{\mathrm{t}}^{0}$, note that these actions are given on both modules by right multiplication on $\mathcal{U}(\mathfrak{k})$ tensored with the adjoint action on the other factor. However, the map $\varphi$ intertwines the adjoint actions of $T$, hence also of $\mathfrak{t}$.

In particular, we see that if $k \geq p$, then for $u \otimes \lambda \in N^{-p}(\mathfrak{k})$ and $n \in N^{-k+p}(\mathfrak{t})$, we have

$$
f^{-k}((u \otimes \lambda) \cdot n)=f^{-p}(u \otimes \lambda) \cdot n,
$$


where $-\cdot n$ denotes the right multiplication by $n$ on $N(\mathfrak{k})$, respectively on the last factor $N(\mathfrak{t})$ in $\left(\mathcal{U}(\mathfrak{k}) \otimes_{\mathbb{C}} \bigwedge^{p}(\mathfrak{k} / \mathfrak{t})[p]\right) \otimes_{\mathcal{U}(\mathfrak{t})} N(\mathfrak{t})$. Note also that $(u \otimes \lambda) \cdot n \in F_{p} N^{-k}(\mathfrak{k})$, so $f^{-k}$ can be applied to it. Using this, let us prove that $f$ is a morphism of complexes.

Let us fix $k>p$; for $k \leq p$ there is nothing to prove. Let $(u \otimes \xi) \eta$ be an element of $F_{p} N^{-k-1}(\mathfrak{k})=N^{-p}(\mathfrak{k}) \bigwedge^{k+1-p}(\mathfrak{t})$. Then

$$
f^{-k}\left(d_{N}((u \otimes \xi) \eta)\right)=f^{-k}\left(d_{N}(u \otimes \xi) \eta+(-1)^{p}(u \otimes \xi) d_{N}(\eta)\right)
$$

by the properties of $d_{N}$. However, $d_{N}(u \otimes \xi) \eta$ is in $F_{p-1} N^{-k}(\mathfrak{k})$ since $\eta \in \bigwedge^{k-p+1}(\mathfrak{t})$, so $f^{-k}$ sends it to 0 . Hence the above expression is equal to

$$
(-1)^{p} f^{-k}\left((u \otimes \xi) d_{N}(\eta)\right)=(-1)^{p} f^{-p}(u \otimes \xi) \cdot d_{N}(\eta) ;
$$

here we used the above remark. Now by 3.2.2(iii),

$$
f^{-p}(u \otimes \xi)=u \otimes \varphi(\xi)=u \otimes \varphi(\xi \cdot 1)=u \otimes \pi^{p}(\xi) \otimes 1,
$$

where $\pi^{p}: \bigwedge^{p}(\mathfrak{k}) \rightarrow \bigwedge^{p}(\mathfrak{k} / \mathfrak{t})$ is induced by the projection $\mathfrak{k} \rightarrow \mathfrak{k} / \mathfrak{t}$. So the above expression is equal to

$(-1)^{p} u \otimes \pi^{p}(\xi) \otimes d_{N}(\eta)=d\left(u \otimes \pi^{p}(\xi) \otimes \eta\right)=d(u \otimes \varphi(\xi \eta))=d\left(f^{-k-1}((u \otimes \xi) \eta)\right)$.

So $f$ is a morphism of complexes and this finishes the proof.

Assume now that $T$ is reductive. Then $\bigwedge^{p}(\mathfrak{k} / \mathfrak{t})$ is a projective $T$-module, and hence $\operatorname{ind}_{\mathcal{U}(\mathfrak{k}), \mathbb{C}} \bigwedge^{p}(\mathfrak{k} / \mathfrak{t})$ is a projective weak $(\mathfrak{k}, T)$-module since $\operatorname{ind}_{\mathcal{U}(\mathfrak{k}), \mathbb{C}}$ is left adjoint to the forgetful functor from $\mathcal{M}(\mathfrak{k}, T)_{w}$ into $\mathcal{M}(T)$. This implies that $\operatorname{ind}_{\mathcal{U}(\mathfrak{k}), \mathbb{C}} \bigwedge^{p}(\mathfrak{k} / \mathfrak{t})[p]$ is a K-projective complex of weak $(\mathfrak{k}, T)$-modules, since it is a translate of $D\left(\operatorname{ind}_{\mathcal{U}(\mathfrak{k}), \mathbb{C}} \bigwedge^{p}(\mathfrak{k} / \mathfrak{t})\right)$, and $D(P)$ is a K-projective complex for any projective object $P$ in an abelian category. Namely, a complex of projectives bounded above is a basic example of a K-projective complex over an abelian category; this is a reformulation of a well-known fact that any morphism between a complex of projectives and an acyclic complex is homotopic to 0. See [Sp] for further discussion.

Now the functor $C_{\mathfrak{t}}$ preserves K-projectives since it is left adjoint to the forgetful functor from equivariant complexes to weak complexes (see 2.5.2(i)). Therefore, by 3.2.4 we see that $G r_{p} N(\mathfrak{k})$ is a $\mathrm{K}$-projective $(\mathfrak{k}, T, N(\mathfrak{t}))$-module. Hence we have proved

3.2.5. Corollary. If $T$ is reductive, then the graded objects $G r_{p} N(\mathfrak{k})$ corresponding to the Hochschild-Serre filtration are K-projective (k, $T, N(\mathfrak{t}))$-modules.

We can now finish the proof of K-projectivity of $N(\mathfrak{k})$ and hence also of 3.1.5.

3.2.6. Theorem. If $T$ is reductive, $N(\mathfrak{k})$ is a K-projective $(\mathfrak{k}, T, N(\mathfrak{t}))$-module.

Proof. By 2.6.4, the only remaining thing to check is that for any $p, F_{p} N(\mathfrak{k})$ is isomorphic to $F_{p-1} N(\mathfrak{k}) \oplus G r_{p} N(\mathfrak{k})$ as a graded $(\mathfrak{k}, T, N(\mathfrak{t}))$-module.

Let $\mathfrak{p}$ be a $T$-invariant complement of $\mathfrak{t}$ in $\mathfrak{k} ; \mathfrak{p}$ exists since $T$ is reductive. Identifying $\mathfrak{k} / \mathfrak{t}$ with $\mathfrak{p}$ gives an identification

$$
G r_{p} N(\mathfrak{k}) \cong\left(\mathcal{U}(\mathfrak{k}) \otimes_{\mathbb{C}} \bigwedge^{p}(\mathfrak{p})\right)[p] \otimes_{\mathbb{C}} \bigwedge(\mathfrak{t})
$$

As a graded module, the last module embeds into $F_{p} N(\mathfrak{k})$ via the inclusion induced by the isomorphism

$$
\bigwedge(\mathfrak{p}) \otimes_{\mathbb{C}} \bigwedge(\mathfrak{t}) \rightarrow \bigwedge(\mathfrak{k})
$$

which is given by multiplication. This inclusion clearly gives the required splitting. 
Now we will tie this with the well-known construction of the relative standard complex used to calculate $(\mathfrak{k}, T)$-cohomology. Note first that it follows from 2.2.6 that the counit morphism

$$
\varepsilon: N(\mathfrak{k}) \rightarrow \mathbb{C},
$$

which is well known to be a resolution of $\mathbb{C}$ by free $\mathcal{U}(\mathfrak{k})$-modules, is also a $\mathrm{K}$ projective resolution of the trivial $(\mathfrak{k}, T, N(\mathfrak{t}))$-module $\mathbb{C}$. Namely, it is obvious that $\varepsilon$ is a $T$-morphism and an $N(\mathfrak{t})$-morphism.

However, $\mathbb{C}$ is actually a $(\mathfrak{k}, T)$-module, and we know from 2.5.2(ii) that the forgetful functor $Q$ from complexes of $(\mathfrak{k}, T)$-modules into $(\mathfrak{k}, T, N(\mathfrak{t}))$-modules has a left adjoint $-\otimes_{N(\mathfrak{t})} \mathbb{C}$, that is, the functor of $N(\mathfrak{t})$-coinvariants. Therefore $\varepsilon$ factors through a morphism

$$
\delta: N(\mathfrak{k}) \otimes_{N(\mathfrak{t})} \mathbb{C} \rightarrow \mathbb{C}
$$

of complexes of $(\mathfrak{k}, T)$-modules. Let us show that $\delta$ is a quasi-isomorphism. Note that $\varepsilon=\delta \circ p$, where $p: N(\mathfrak{k}) \rightarrow N(\mathfrak{k}) \otimes_{N(\mathfrak{t})} \mathbb{C}$ is given by $n \mapsto n \otimes 1 ; p$ is actually the adjunction morphism. Therefore it is enough to show that $p$ is a quasi-isomorphism. However, if we identify $N(\mathfrak{k})$ with $N(\mathfrak{k}) \otimes_{N(\mathfrak{t})} N(\mathfrak{t})$, then $p$ gets identified with

$$
1 \otimes \varepsilon^{\prime}: N(\mathfrak{k}) \otimes_{N(\mathfrak{t})} N(\mathfrak{t}) \rightarrow N(\mathfrak{k}) \otimes_{N(\mathfrak{t})} \mathbb{C},
$$

where $\varepsilon^{\prime}: N(\mathfrak{t}) \rightarrow \mathbb{C}$ is the counit morphism, which is again a quasi-isomorphism. By Poincaré-Birkhoff-Witt Theorem (2.2.1), $N(\mathfrak{k})$ is a free right $N(\mathfrak{t})$-module for the right multiplication. Therefore, by [BL1], 10.12.4.4, it is a K-flat $N(\mathfrak{t})$-module and it follows that $1 \otimes \varepsilon^{\prime}$ is a quasi-isomorphism.

On the other hand, since the functor $-\otimes_{N(\mathfrak{t})} \mathbb{C}$ preserves K-projectives, $N(\mathfrak{k}) \otimes_{N(\mathfrak{t})}$ $\mathbb{C}$ is a $\mathrm{K}$-projective complex of $(\mathfrak{k}, T)$-modules. In fact, its components are easily seen to be

$$
\mathcal{U}(\mathfrak{k}) \otimes_{\mathcal{U}(\mathfrak{t})} \bigwedge^{i}(\mathfrak{p}),
$$

so they are projective $(\mathfrak{k}, T)$-modules. Namely, $\bigwedge^{i}(\mathfrak{p})$ are projective $T$-modules, that is, projective $(\mathfrak{t}, T)$-modules, and the above modules are $\operatorname{ind}_{\mathcal{U}(\mathfrak{k}), \mathcal{U}(\mathfrak{t})} \wedge^{i}(\mathfrak{p})$, so they are projective by 1.2.3.

Note that the above modules are also finitely generated $\mathcal{U}(\mathfrak{k})$-modules. To conclude

3.2.7. Proposition. If $T$ is reductive, then the morphism

$$
N(\mathfrak{k}) \otimes_{N(\mathfrak{t})} \mathbb{C} \rightarrow \mathbb{C}
$$

constructed above is a resolution of the trivial $(\mathfrak{k}, T)$-module $\mathbb{C}$ by projective $(\mathfrak{k}, T)$ modules, which are finitely generated as $\mathcal{U}(\mathfrak{k})$-modules.

Of course, this is just the well-known relative standard complex (see e.g. [BW]).

3.3. Equivariant versus classical Zuckerman functors. In this section we relate the equivariant construction from Section 3.1 to the classical Zuckerman construction. Let $V$ be an $(\mathcal{A}, T)$-module. Let $D(V)$ be the corresponding complex of $(\mathcal{A}, T)$-modules (concentrated in degree 0$)$. Consider the corresponding $(\mathcal{A}, T, N(\mathfrak{t}))$-module $Q(D(V))$ ( $Q$ is as in 2.5.2(ii)). This is again a complex concentrated in degree 0 and having $V$ as the $0^{\text {th }}$ component; the actions of $\mathcal{A}$ and $T$ are the same as on $V$, while the action of $N(\mathfrak{t})$ is trivial. Let us consider the module $R(K, Q(D(V)))$. As a complex of weak $(\mathcal{A}, K)$-modules, with actions $\pi_{R}$ 
and $\nu_{R}$, it is just $D(R(K, V))$. As a $(\mathfrak{k}, T, N(\mathfrak{t}))$-module, with action $\lambda$, it must be equal to $Q(D(R(K, V)))$ since it is concentrated in degree 0 . It follows that

$$
\Gamma_{K, T}^{e q}(Q(D(V)))=\operatorname{Hom}_{(\mathfrak{k}, T, N(\mathfrak{t}))}(N(\mathfrak{k}), Q(D(R(K, V)))) .
$$

As we already mentioned, by 2.5.2(ii), $Q: K(\mathcal{M}(\mathfrak{k}, T)) \rightarrow K(\mathfrak{k}, T, N(\mathfrak{t}))$ has a left adjoint, the functor $-\otimes_{N(\mathfrak{t})} \mathbb{C}$ of $N(\mathfrak{t})$-coinvariants. Since both these functors satisfy the conditions of 2.4.5, the stronger variant of adjunction from 2.4.5 holds, i.e., we have

$$
\operatorname{Hom}_{(\mathfrak{k}, T, N(\mathfrak{t}))}(N(\mathfrak{k}), Q(D(R(K, V))))=\operatorname{Hom}_{(\mathfrak{k}, T)}\left(N(\mathfrak{k}) \otimes_{N(\mathfrak{t})} \mathbb{C}, D(R(K, V))\right) .
$$

The actions of $\mathcal{A}, K$ and $N(\mathfrak{k})$ are given on the right-hand side in the same way as on the left-hand side: $\mathcal{A}$ and $K$ act on the second variable, while $N(\mathfrak{k})$ acts on the first (by left multiplication). With this definition, it is obvious that the above equality is an equality of $(\mathcal{A}, K, N(\mathfrak{k}))$-modules.

However, as we saw in $3.2 .7, N(\mathfrak{k}) \otimes_{N(\mathfrak{t})} \mathbb{C}$ is a projective resolution of the trivial module $\mathbb{C}$ in the category of $(\mathfrak{k}, T)$-modules. It follows that as a complex of weak $(\mathcal{A}, K)$-modules,

$$
\Gamma_{K, T}^{e q}(Q(D(V)))=R \operatorname{Hom}_{(\mathfrak{k}, T)}(D(\mathbb{C}), D(R(K, V))) .
$$

In particular, we get

3.3.1. Lemma. Let $V$ be an $(\mathcal{A}, T)$-module. If $T$ is reductive, then the cohomology modules of $\Gamma_{K, T}^{e q}(Q(D(V)))$ are given by

$$
H^{p}\left(\Gamma_{K, T}^{e q}(Q(D(V)))\right)=\operatorname{Ext}_{(\mathfrak{k}, T)}^{p}(\mathbb{C}, R(K, V))
$$

as $(\mathcal{A}, K)$-modules. Here $R(K, V)$ is considered as a $(\mathfrak{k}, T)$-module with respect to the $\lambda$-action.

Using 1.3.3, we finally get

3.3.2. Theorem. Assume that $T$ is reductive and that $\mathcal{A}$ is a flat $\mathcal{U}(\mathfrak{k})$-module for the right multiplication (for example, this is true for $\mathcal{A}=\mathcal{U}(\mathfrak{g})$, where $(\mathfrak{g}, K)$ is a Harish-Chandra pair). Let $V$ be an $(\mathcal{A}, T)$-module. Then the cohomology modules of $\Gamma_{K, T}^{e q}(Q(D(V)))$ are equal to the classical derived Zuckerman functors of $V$.

\section{REFERENCES}

[BB] A. Beilinson, J. Bernstein, A proof of the Jantzen conjecture, (preprint), M.I.T. and Harvard University (1989).

[BL1] J. Bernstein, V. Lunts, Equivariant sheaves and functors, Lecture Notes in Math., vol. 1578, Springer-Verlag, 1994. MR.1299527 (95k:55012)

[BL2] J. Bernstein, V. Lunts, Localization for derived categories of $(\mathfrak{g}, K)$-modules, J. Amer. Math. Soc. 8 No. 4 (1995), 819-856. MR.1317229 (95m:17004)

[BW] A. Borel, N. Wallach, Continuous cohomology, discrete subgroups, and representations of reductive groups, Annals of Math. Studies, vol. 94, Princeton University Press, Princeton, 1980. MR0554917 (83c:22018)

[Bo] N. Bourbaki, Algèbre, Ch. 1 à 3, Bourbaki, Paris, 1970. MR0274237 (43:2)

[De] P. Deligne, Cohomologie à supports propres, SGA 4, Lecture Notes in Math., vol. 305, Springer-Verlag, Berlin, Heidelberg, 1973. MR0354654 (50:7132)

[DV] M. Duflo, M. Vergne, Sur les functeurs de Zuckerman, C. R. Acad. Sci. Paris 304 (1987), 467-469. MR0894570 (89h:22025)

[EW] T.J. Enright, N.R. Wallach, Notes on homological algebra and representations of Lie algebras, Duke Math. J. 47 (1980), 1-15. MR0563362 (81c:17013)

[GM] S. I. Gelfand, Yu.I. Manin, Methods of homological algebra, Springer-Verlag, Berlin, Heidelberg, New York, 1996. MR1438306 (97j:18001) 
[G] V. A. Ginzburg, Equivariant cohomology and Kähler geometry, (Russian), Funktsional. Anal. i Prilozhen. 21 no. 4 (1987), 19-34. MR0925070 (89b:58013)

[HMSW] H. Hecht, D. Miličić, W. Schmid, J.A. Wolf, Localization and standard modules for real semisimple Lie groups I: The duality theorem, Inventiones Math. 90 (1987), 297-332. MR0910203 (89e:22025)

[HS] G. Hochschild, J.P. Serre, Cohomology of Lie algebras, Annals of Math. 57 (1953), 591-603. MR0054581 (14:943c)

[Il] L. Illusie, Complexe cotangent et déformations I, Lecture Notes in Math., vol. 239, 1971; II, Lecture Notes in Math., vol. 283, Springer-Verlag, Berlin, Heidelberg, 1972. MR0491680 (58:10886a) MR0491681 (58:10886b)

[KS] M. Kashiwara, P. Schapira, Sheaves on manifolds, Springer-Verlag, Berlin, Heidelberg, 1990. MR1074006 (92a:58132)

[KV] A.W. Knapp, D.A. Vogan, Cohomological induction and unitary representations, Princeton University Press, Princeton, 1995. MR.1330919 (96c:22023)

$[\mathrm{M}] \quad$ D. Miličić, Lectures on derived categories, http://www.math.utah.edu/ $\sim$ milicic/ dercat.pdf.

[MP1] D. Miličić, P. Pandžić, Equivariant derived categories, Zuckerman functors and localization, Geometry and representation theory of real and $p$-adic Lie groups (J. Tirao, D. Vogan, J. A. Wolf, eds.), Progress in Mathematics 158, Birkhäuser, Boston, 1996, pp. 209-242. MR.1486143 (2000f:22018)

[MP2] D. Miličić, P. Pandžić, Cohomology of standard Harish-Chandra sheaves, (in preparation), University of Utah and University of Zagreb.

[P1] P. Pandžić, Equivariant analogues of Zuckerman functors, Ph.D. thesis, University of Utah, 1995.

[P2] P. Pandžić, A simple proof of Bernstein-Lunts equivalence, Manuscripta Math. 118 (2005), 71-84. MR2171292

[Sch] M. Schenuert, The theory of Lie superalgebras, Lecture Notes in Math., vol. 716, Springer-Verlag, Berlin, Heidelberg, 1979. MR0537441 (80i:17005)

[Sp] N. Spaltenstein, Resolutions of unbounded complexes, Compositio Math. 65 (1988), 121-154. MR0932640 (89m:18013)

[Ve1] J.L. Verdier, Catégories dérivées, état 0, SGA 4 $\frac{1}{2}$, Lecture Notes in Math., vol. 569, Springer-Verlag, 1977. MR0463174 (57:3132)

[Ve2] J.L. Verdier, Des catégories dérivées des catégories abéliennes, with a preface by Luc Illusie; edited and with a note by Georges Maltsiniotis, Astérisque 239 (1996). MR.1453167 (98c:18007)

[Vo] D.A. Vogan, Representations of real reductive Lie groups, Birkhäuser, Boston, 1981. MR0632407 (83c:22022)

[VZ] D.A. Vogan, G.J. Zuckerman, Unitary representations with non-zero cohomology, Compositio Math. 53 (1984), 51-90. MR0762307 (86k:22040)

[W] N.R. Wallach, Real reductive Groups I, Academic Press, Boston, 1988. MR0929683 (89i:22029)

[Z] G. J. Zuckerman, Lecture Series "Construction of representations via derived functors", Institute for Advanced Study, Princeton, N.J., Jan.-Mar., 1978.

Department of Mathematics, University of Zagreb, PP 335, 10002 Zagreb, Croatia

E-mail address: pandzic@math.hr 